\title{
Peripheral blood CD4+CCR6+ compartment differentiates HIV-1 infected or seropositive elite controllers from long-term successfully treated individuals
}

Sara Svensson Akusjärvi ( $\nabla$ sara.svensson.akusjarvi@ki.se )

Karolinska Institutet https://orcid.org/0000-0002-1086-5409

Shuba Krishnan

Karolinska Institute

Bianca Jütte

Karolinska Institutet

Anoop Ambikan

Karolinska Institute

Soham Gupta

Karolinska Institute https://orcid.org/0000-0003-1136-3010

Jimmy Rodriguez

Karolinska Institutet https://orcid.org/0000-0002-6735-3332

Akos Vegvari

Karolinska Institute https://orcid.org/0000-0002-1287-0906

Maike Sperk

Karolinska Institute

Piotr Nowak

Karolinska University Hospital

Jan Vesterbacka

Karolinska Institutet

\section{J. Peter Svensson}

Karolinska Institutet https://orcid.org/0000-0002-5863-6250

Anders Sonnerborg

Karolinska Institutet

Ujjwal Neogi

Karolinska Institutet https://orcid.org/0000-0002-0844-3338 
Keywords: PLWH, HIV-1, PLWHART, ART, CCR5, sterilising cure

Posted Date: November 17th, 2021

DOI: https://doi.org/10.21203/rs.3.rs-956654/v1

License: (c) (1) This work is licensed under a Creative Commons Attribution 4.0 International License. Read Full License

Version of Record: A version of this preprint was published at Communications Biology on April 13th, 2022. See the published version at https://doi.org/10.1038/s42003-022-03315-x. 
1 Peripheral blood $\mathrm{CD}^{+} \mathrm{CCR6}^{+}$compartment differentiates HIV-1 infected or seropositive 2 elite controllers from long-term successfully treated individuals

3

4

\section{Sara Svensson Akusjärvi ${ }^{1, *}$, Shuba Krishnan ${ }^{1}$, Bianca B. Jütte ${ }^{2}$, Anoop T. Ambikan ${ }^{1}$, Soham Gupta ${ }^{1}$,} Jimmy Esneider Rodriguez ${ }^{3}$, Ákos Végvári ${ }^{3}$, Maike Sperk ${ }^{1}$, Piotr Nowak ${ }^{4}$, Jan Vesterbacka ${ }^{4}$, J. Peter Svensson $^{2}$, Anders Sönnerborg ${ }^{1,4}$, and Ujjwal Neogi ${ }^{1,5,6, *}$

\section{Affiliations}

${ }^{1}$ Division of Clinical Microbiology, Department of Laboratory Medicine, Karolinska Institutet, ANA Futura, Campus Flemingsberg, 14152 Stockholm, Sweden

${ }^{2}$ Department of Biosciences and Nutrition, Karolinska Institutet, Neo, Campus Flemingsberg, 14183 Stockholm, Sweden

${ }^{3}$ Division of Chemistry I, Department of Medical Biochemistry and Biophysics, Karolinska Institutet, Campus Solna, 17165 Stockholm, Sweden

${ }^{4}$ Division of Infectious Disease, Department of Medicine Huddinge, Karolinska Institutet, I73, Karolinska University Hospital, 14186 Stockholm, Sweden

${ }^{5}$ Christopher S. Bond Life Sciences Centre, University of Missouri, Columbia, MO, 65211, USA

${ }^{6}$ Manipal Institute of Virology (MIV), Manipal Academy of Higher Education, Manipal, Karnataka, India

${ }^{*}$ Corresponding Authors: Sara Svensson Akusjärvi (sara.svensson.akusjarvi@ki.se) ; Ujjwal Neogi (ujjwal.neogi@ki.se)

\section{Abstract}

HIV-1 infection induces a chronic inflammatory environment not restored by suppressive antiretroviral therapy (ART). As of today, the effect of viral suppression and immune reconstitution in people living with HIV-1 (PLWH) on long-term suppressive ART ( PLWH $_{\mathrm{ART}}$ ) is not well-described. Herein, we show how PLWH who naturally control the virus $\left(\mathrm{PLWH}_{\mathrm{EC}}\right)$ have a reduced proportion of $\mathrm{CD} 4^{+} \mathrm{CCR} 6^{+}$cells compared to $\mathrm{PLWH}_{\mathrm{ART}}$ and HIV-1 negative controls. Specifically, the $\mathrm{CD} 4^{+} \mathrm{CCR} 6^{+} / \mathrm{CCR} 6^{-}$cells exhibited a uniqe proteomic profile with a modulated energy metabolism in $\mathrm{PLWH}_{\mathrm{EC}}$ compared to $\mathrm{PLWH}_{\mathrm{ART}}$ independent of CCR6 status. Furthermore, the $\mathrm{CD} 4^{+} \mathrm{CCR} 6^{+}$cells were enriched in proteins involved in apoptosis and $\mathrm{p} 53$ signalling in $\mathrm{PLWH}_{\mathrm{EC}}$ compared to $\mathrm{PLWH}_{\mathrm{ART}}$, indicative of increased sensitivity towards cell death mechanisms. Collectively, this data shows how $\mathrm{PLWH}_{\mathrm{EC}}$ have a unique chemokine receptor profile that may aid in facilitating natural control of HIV-1 infection. 
Efficient antiretroviral therapy (ART) supresses human immunodeficiency virus type 1 (HIV-1) replication to below the detection level of sensitive HIV RNA assays. Still, HIV-1 persists for decades despite efficient ART in presumably latently infected cells, making up the so-called latent viral reservoir. This reservoir resides in subtypes of memory $\mathrm{T}$ cells and cells from monocytic lineages, but also in some functional and naïve $\mathrm{T}$ cells subsets ${ }^{1}$. Eradication of latently infected cells, i.e. a sterilizing cure, has proven to be a major hurdle due to several factors such as heterogeneity in mechanisms governing latency and integration sites into the host genome ${ }^{2}$. Over the course of suppressive ART, persisting HIV-1 sustained by low levels of viral replication, homeostatic proliferation, and cell to cell transmission, is a driver of chronic immune activation in the host ${ }^{3,4,5}$. In HIV-1 infection, chemokine receptors are essential co-receptors needed for HIV-1 entry into the cell where the CC chemokine receptor 5 (CCR5) and CXCR4 are the main co-receptors used for HIV-1 entry, but alternative receptors such as CCR3 may play a role for macrophage tropic viral strains $6,7,8$. $\mathrm{T}$ cells expressing the chemokine receptor CCR6 are also overrepresented amongst cell types infected by HIV-1 ${ }^{9}$. Furthermore, $\mathrm{CD} 4^{+} \mathrm{CCR} 6^{+}$and $\mathrm{CD} 4^{+} \mathrm{CCR} 2^{+} \mathrm{CCR} 5^{+}$cells have been proposed to contribute to the HIV-1 reservoir ${ }^{10,11}$.

Alterations in chemokine receptor expression and chemokine levels modulate the activity of the immune response and inflammatory levels during HIV-1 infection ${ }^{12}$. In people living with HIV-1 (PLWH) on suppressive ART, these aberrations may contribute to i) higher risk of age associated co-morbidities together with elevated immune activation ii) microbiome dysbiosis leading to microbial translocation and iii) dysregulated immune cell activation and function ${ }^{13,14,15,16}$. Consequently, HIV-1 pathogenesis and immune dysfunction promote chronic immune activation. This results in a vicious cycle as immune activation likely drives HIV-1 disease progression and inflammaging, defined as an age-related increase of inflammatory markers, that could possibly lead to earlier onset of age-related diseases. In our earlier study on a small group of 10 PLWH on ART for two decades, we reported normalisation of most of the plasma proinflammatory cytokines and chemokines to the level of HIV-negative controls ${ }^{17}$. Therefore, although there are several studies on immune reconstitution and viral suppression during short-term ART, the long-term effect of ART on the immune system is not known.

Eradication of latently infected cells, i.e. a sterilising cure, has proven to be a major hurdle. An alternative approach is a functional cure aimed at suppressing viral replication without ART. A model for functional cure studies is elite controllers (herein $\mathrm{PLWH}_{\mathrm{EC}}$ ). $\mathrm{PLWH}_{\mathrm{EC}}$ constitute a small fraction $(<0.5 \%)$ of $\mathrm{HIV}-1^{+}$individuals able to naturally control viral replication in the absence of ART ${ }^{18,19}$. However, there is a population-based heterogeneity of how these individuals supress viral replication which is likely caused by several factors such as viral genetic factors, variability of integration site in the human genome, variation in the host response, and immunological factors ${ }^{20,21,22}{ }^{23}$. In terms of immune cell activation, studies have shown how $\mathrm{PLWH}_{\mathrm{EC}}$ maintain lower levels of inflammatory 
markers compared to PLWH who progress in their disease ${ }^{24}$. However, as no consensus exists on how the $\mathrm{PLWH}_{\mathrm{EC}}$ phenotype is maintained, further studies comparing variability of immune function between PLWH $\mathrm{EC}_{\mathrm{EC}}$ and PLWH on suppressive ART (herein $\mathrm{PLWH}_{\mathrm{ART}}$ ) are required to understand the mechanisms of natural control of HIV-1.

In our recent study ${ }^{25}$, we hypothesised that a modulated CCR6/CCL20 chemokine axis and CCR2CCL7-CCL2 signalling may play a protective role in the PLWHEC phenotype. We identified a downregulation of CCR2 and CCR6 receptors on $\mathrm{CD}^{+}$and $\mathrm{CD} 8^{+}$lymphocytes and higher plasma abundance of CCL4, CCL7 and CCL20 in PLWHEC compared to the HIV-1-negative controls. This can provide natural resistance to HIV-1 infection although the expression profile during long-term suppressive ART is not known. In the present study, we extended our analysis to evaluate the expression

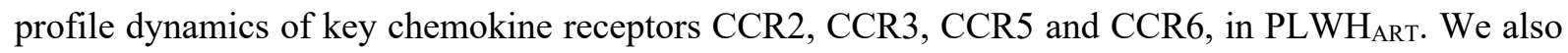

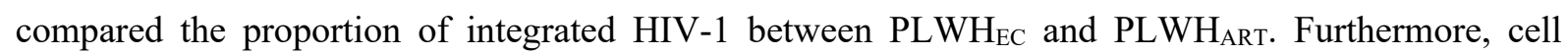
populations of interest were isolated for quantitative proteomics to evaluate specific characteristics regulating these cell populations and their potential role in HIV-1 persistence. Our study provides important understanding of the chemokine receptor dynamics and its role in HIV-1 persistence that differentiate $\mathrm{PLWH}_{\mathrm{EC}}$ from PLWH $\mathrm{PRT}_{\mathrm{AR}}$.

\section{Results}

$\mathrm{PLWH}_{\mathrm{EC}}$ have reduced populations of $\mathrm{CD4}^{+} \mathrm{CCR6}^{+}$and $\mathrm{CD4}^{+} \mathrm{CCR2}^{+} \mathrm{T}$ lymphocytes compared to successfully long-term treated patients

Herein, we wanted to evaluate if there are any differences in the expression profile of some key chemokine receptors between PLWH with natural control of the virus and those on ART. Therefore, we investigated the expression levels of CCR2, CCR3, CCR5, and CCR6 in peripheral blood mononuclear cells (PBMCs) from PLWH $\mathrm{EC}_{(}(n=14), \mathrm{PLWH}_{\mathrm{ART}}(n=54)$, and HIV negative individuals (HC, $\left.n=18\right)$ by flow cytometry (Supplementary Fig 1). The clinical and demographic data are presented in Table 1 and Supplementary Table 1. All the PLWH $\mathrm{ART}_{\mathrm{A} T}$ were successfully treated at the time of sample collection and plasma viral load was below the detection level $(<40$ copies $/ \mathrm{mL})$. Initial discrimination between $\mathrm{CD}^{+} \mathrm{T}$ cells $\left(\mathrm{CD}^{+} \mathrm{CD}^{+}\right)$and $\mathrm{CD} 8^{+} \mathrm{T}$ cells $\left(\mathrm{CD}^{+} \mathrm{CD}^{+}\right)$showed that both $\mathrm{PLWH}_{\mathrm{EC}}$ and $\mathrm{PLWH}_{\mathrm{ART}}$ exhibited a reduced proportion of $\mathrm{CD}^{+} \mathrm{T}$ cells and elevated proportion of $\mathrm{CD}^{+} \mathrm{T}$ cells compared to HC (Fig. 1a). Single receptor expression in $\mathrm{PLWH}_{\mathrm{EC}}$ showed a distinct profile compared to both PLWH $\mathrm{ART}_{\mathrm{A} T}$ and $\mathrm{HC}$ (Fig. 1b). Expression of CCR2 and CCR6 was reduced on both $\mathrm{CD}^{+}$and $\mathrm{CD} 8^{+} \mathrm{T}$ cells together with a decreased proportion of $\mathrm{CD} 8^{+} \mathrm{CCR} 2^{+}, \mathrm{CD} 4^{+} \mathrm{CCR} 6^{+}$, and $\mathrm{CD} 8^{+} \mathrm{CCR} 6^{+}$in PLWHEC $_{\mathrm{EC}}$ compared to both PLWH $\mathrm{ART}_{\mathrm{A}}$ and HC (Fig. 1c, d and Supplementary Fig. 2a and b). The expression levels of CCR2 were also reduced on $\mathrm{CD}^{+} \mathrm{T}$ cells in $\mathrm{PLWH}_{\mathrm{ART}}$ compared to HC, as well as the proportion of $\mathrm{CD} 8^{+} \mathrm{CCR} 6^{+}$cells (Fig. 1c, and Supplementary Fig. 2b). Even as the total frequency of $\mathrm{CD}^{+} \mathrm{CCR}^{+}$was low in all three groups, the expression of CCR3 was high in both $\mathrm{PLWH}_{\mathrm{EC}}$ and 
PLWH $_{\text {ART }}$ compared to HC (Supplementary Fig. 2c). Furthermore, expression levels of CCR5 were lower on $\mathrm{CD}^{+} \mathrm{T}$ cells in PLWHEC compared to $\mathrm{PLWH}_{\mathrm{ART}}$ (Supplementary Fig. 2d), while no

111 difference was detected on $\mathrm{CD}^{+} \mathrm{T}$ cells. Collectively, these data show how $\mathrm{PLWH} \mathrm{EC}_{\mathrm{EC}}$ have a unique 112 expression profile of CCR2 and CCR6 while the expression signature of CCR3 is more similar to $113 \mathrm{PLWH}_{\mathrm{ART}}$ on lymphocytic cells (Fig. 1e). Continuing, co-expression analysis on $\mathrm{CD}^{+}$cells showed 114 that $\mathrm{CCR} 2^{+} \mathrm{CCR} 5^{+} \mathrm{CCR} 6^{+}, \mathrm{CCR} 2^{+} \mathrm{CCR} 6^{+}$, and $\mathrm{CCR} 5^{+} \mathrm{CCR} 6^{+}$were reduced in $\mathrm{PLWH}_{\mathrm{EC}}$ compared to 115 both PLWH ART $_{\text {and }}$ HC, while no differences were detected between PLWH ART $_{\text {and }}$ HC (Fig. 1f). 116 Furthermore, single receptor expression of CCR2 was reduced in PLWH $\mathrm{EC}_{\mathrm{EC}}$ compared to PLWH $\mathrm{ART}_{\mathrm{And}}$ 117 CCR6 expression was reduced in $\mathrm{PLWH}_{\mathrm{EC}}$ compared to both $\mathrm{PLWH}_{\mathrm{ART}}$ and $\mathrm{HC}$ (Fig. 1f). Within the $118 \mathrm{CD}^{+} \mathrm{T}$ cell population, co-receptor expression of $\mathrm{CCR} 2^{+} \mathrm{CCR} 5^{+} \mathrm{CCR} 6^{+}$was reduced in both $\mathrm{PLWH}_{\mathrm{ART}}$ 119 and $\mathrm{PLWH} \mathrm{EC}_{\mathrm{EC}}$ compared to $\mathrm{HC}$ while CCR2 ${ }^{+} \mathrm{CCR} 5^{+}$was reduced in $\mathrm{PLWH}_{\mathrm{EC}}$ compared to $\mathrm{HC}$ and $120 \mathrm{PLWH}_{\mathrm{ART}}$ (Fig. 1g). Single receptor expression analysis also showed that CCR5 was increased in $121 \mathrm{PLWH}_{\mathrm{EC}}$ compared to HC and CCR6 expression increased in PLWHEC compared to both HC and PLWH $_{\text {ART }}$ (Fig. 1g). As the major differences were detected on CCR2 and CCR6 expression, we measured the levels of their ligands, namely monocyte chemoattractant protein 1, MCP1 (CCL2) and macrophage inflammatory protein 3, MIP-3 (CCL20) respectively, in plasma from PLWH $\operatorname{ART}_{\text {A }}(n=51)$, PLWHEC $(n=12)$, and HC $(n=17)$. Plasma levels of CCL20 were increased in both PLWHEC and PLWH $_{\text {ART }}$ compared to HC, while no significant difference was detected for CCL2 (Supplementary Fig. 2e and f). In summary, the receptor expression profile on lymphocytes in $\mathrm{PLWH}_{\mathrm{ART}}$ resembles $\mathrm{HC}$, while $\mathrm{PLWH}_{\mathrm{EC}}$ exhibits a distinct expression profile with decreased receptor expression of both CCR2 and CCR6.

\section{CCR2 expression is reduced in $\mathrm{PLWH}_{\mathrm{EC}}$ compared to $\mathrm{PLWH}_{\mathrm{ART}}$ on monocytic cell populations} In HIV-1 infection there is a chronic activation of monocytes contributing to organ specific inflammatory events that are poorly understood in virologically suppressed patients. Our group and others have previously shown persistent low-level inflammation in $\mathrm{PLWH}_{\mathrm{EC}}{ }^{24,25,26}$. Furthermore, chemokine receptors like CCR2 and CCR5 play a major role in inflammatory trafficking of monocytes ${ }^{27}$. Therefore, we determined the CCR2, CCR3, CCR5, and CCR6 receptor expression on the monocytic cell populations; classical $\left(\mathrm{CM}, \mathrm{CD} 14^{+} \mathrm{CD} 16^{-}\right)$, intermediate $\left(\mathrm{IM}, \mathrm{CD} 14^{+} \mathrm{CD} 16^{+}\right)$, and non-classical $\left(\mathrm{NCM}, \mathrm{CD} 14^{-} \mathrm{CD} 16^{+}\right)$. Herein, $\mathrm{PLWH}_{\mathrm{EC}}$ exhibited reduced numbers of $\mathrm{CM}$ compared to $\mathrm{HC}$ and

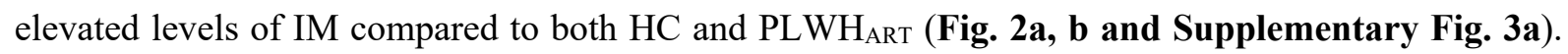
Generally, the receptor expression of CCR3 and CCR6 was low on all three monocytic cell subsets (Fig. 2c). The major difference was a reduced expression of CCR2 on CM and IM in $\mathrm{PLWH}_{\mathrm{EC}}$ compared to

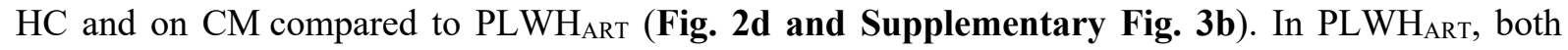

143 frequency of IM-CCR2 ${ }^{+}$cells and expression of CCR2 on IM cells were reduced compared to HC (Fig.

$1442 d$ and Supplementary Fig. 3b). Although the proportion of CM-CCR6 ${ }^{+}$cells was decreased in

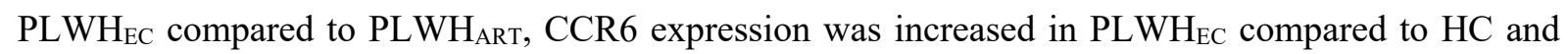


$146 \mathrm{PLWH}_{\mathrm{ART}}$, while PLWH $\mathrm{ART}_{\mathrm{ART}}$ showed reduced expression compared to HC (Supplementary Fig. 3c).

147 Furthermore, NCM-CCR6 $6^{+}$cells were lower in PLWHEC compared to PLWH $\mathrm{ART}_{\mathrm{ART}}$ and $\mathrm{HC}$, and the

148 frequency of CM-CCR3 ${ }^{+}$cells was decreased in PLWHEC compared to PLWH $_{\mathrm{ART}}$ (Supplementary Fig.

149 3c and d). The receptor expression of CCR5 was reduced on IM in PLWH ${ }_{A R T}$ compared to HC and on

150 NCM in PLWHEC compared to both PLWH $\mathrm{ART}_{\mathrm{ET}}$ and HC (Supplementary Fig. 3e). This data indicates

151 that the main difference on the monocytic cell subsets is a reduced expression of CCR2 in PLWH $\mathrm{EC}_{\mathrm{EC}}$

152 compared to the other groups (Fig. 2e). The IM were the only category of cells which exhibited

153 differences in co-receptor expression. For these cells, the largest variation was a reduction of

$154 \mathrm{CCR}^{+} \mathrm{CCR}^{+}$expressing cells in both PLWH $\mathrm{EC}_{\mathrm{EC}}$ and PLWH $\mathrm{ART}_{\mathrm{A}}$ compared to HC. An increase in cells

155 lacking all four receptors was found in both $\mathrm{PLWH}_{\mathrm{EC}}$ and $\mathrm{PLWH}_{\mathrm{ART}}$ compared to $\mathrm{HC}$ (Fig. 2f).

156 Furthermore, PLWH $\mathrm{ART}_{\mathrm{ART}}$ exhibited reduced numbers of cells expressing only CCR2 compared to HC

157 (Fig. 2f). Collectively, this data shows that the largest variation occurred on IM where PLWHART and

158 PLWHEC $_{\mathrm{EC}}$ showed a similar trend compared to HC.

159

\section{Longer suppressive therapy reduces expression of CCR2 on monocytes}

161 The chronic inflammatory condition due to HIV-1 persistence and an activated immune response 162 persists during suppressive ART. Therefore, to evaluate if longer treatment duration affects the receptor 163 expression, we included longitudinal samples (timepoint 1 (T1) $n=10$ and timepoint 2 (T2) $n=10$ ) where 164 the same patients were followed-up after three years of additional suppressive therapy (clinical 165 parameters in Supplementary Table 2). Treatment duration did not affect frequencies of CD4 ${ }^{+}, \mathrm{CD}^{+}$

$166 \mathrm{~T}$ cells or monocytic cell populations (Supplementary Fig. 4a and b). For the detected receptor 167 expressions, most of the differences were detected in the monocytic cell populations while no significant 168 variations were seen on $\mathrm{CD}^{+} \mathrm{T}$ cells (Fig. 3a). Longer treatment duration increased the frequency of $169 \mathrm{CD}^{+} \mathrm{CCR} 2^{+}$cells, while all three monocytic cell populations exhibited reduced numbers of cells expressing CCR2 in T2 (Fig. 3b and c). Following a similar trend, CCR2 expression was increased on $\mathrm{CD}^{+} \mathrm{T}$ cells and IM exhibited reduced expression of CCR2 in T2 while CM and NCM showed no difference (Fig. 3d). The proportion of $\mathrm{CD}^{+} \mathrm{CCR}^{+}$cells was increased in $\mathrm{T} 2$, without showing any significant effect of CCR2 expression levels (Fig. 3e-g). Furthermore, the proportions of IM-CCR $3^{+}$and

174 IM-CCR5 ${ }^{+}$cells decreased over time while only expression levels of CCR5 decreased in T2 (Fig. $3 \mathbf{h}$ -

175 m). Investigations of co-receptor expression on the lymphocytic cell populations showed that the only 176 difference was an increase in $\mathrm{CCR} 2^{+} \mathrm{CCR} 5^{+}$in $\mathrm{T} 2$ on $\mathrm{CD} 8^{+} \mathrm{T}$ cells (Fig. 3n). Within the monocytic cell populations, the only differences of co-expression of the receptors were seen on IM where T2 exhibited higher percentage of cells negative for all four receptors while $\mathrm{CCR} 2^{+} \mathrm{CCR} 3^{+} \mathrm{CCR} 5^{+}, \mathrm{CCR} 2^{+} \mathrm{CCR} 3^{+}$, and $\mathrm{CCR} 2{ }^{+} \mathrm{CCR} 5^{+}$were decreased in $\mathrm{T} 2$ (Fig. 3o). Conclusively, the effect induced by longer suppressive treatment mainly reduces expression levels of these chemokine receptors on IM. 
183 To evaluate the proportion of HIV-1 infected cells in our cohort, we quantified total HIV-1 DNA in the 184 PLWH $_{\mathrm{EC}}(n=14)$ and PLWH ${ }_{\mathrm{ART}}(n=54)$. Herein, we observed a higher level of total HIV-1 DNA in 185 PLWH $_{\text {ART }}$ compared to PLWHEC (Fig. 4a) despite a median of eight years of successful treatment. No 186 significant difference was observed between long term ART (lnART; >10yrs (median 20yrs, 187 Interquartile range (IQR); 18-22.75), $n=20$ ) and short-term ART (sART; <10yrs (median $7 \mathrm{yrs,} \mathrm{IQR;} \mathrm{6-}$ 188 8), $n=34$ ) nor was the reservoir affected by longer suppressive treatment between T1 and T2 189 (Supplementary Fig. 5a and b). This data indicates that after the initial two phases of HIV DNA decline 190 the size of the reservoir is not affected during successful suppressive ART. However, PLWHEC exhibited 191 reduced proportions of HIV-1 infected cells presumably due to their natural control of HIV-1. Next, to 192 evaluate the proportion of integrated HIV-1 that was intact/defective, we employed the intact provirus 193 DNA assay (IPDA) ${ }^{28}$ together with quantification of the 5'LTR and 2-LTR circles on the genomic DNA

194 from PBMCs. A significant increase of 3'deletion/hypermutation was detected in PLWH $_{\text {ART }}$ compared

195 to PLWHEC, but no significant differences in intact provirus, 5'deletion, 5'LTR, or 2-LTR circles 196 between the two groups (Fig. 4b). Moreover, percentages of intact provirus out of total HIV-1 DNA did not differ between the groups (Supplementary Fig. 5c). The CCL20 ligand for CCR6 can facilitate nuclear integration of HIV-1 by remodelling the actin cytoskeleton, but also directly inhibits infection in the female reproductive tract ${ }^{29}{ }^{30}$. Therefore, to evaluate if the ligands for CCR2 and CCR6 might have an effect on the HIV-1 reservoir we performed correlation analysis between plasma levels of CCL20 and CCL2 and the HIV-1 reservoir. No significant correlation between HIV-1 DNA and CCL20 plasma levels was revealed in either PLWH ART $_{\text {or }}$ PLWHEC $_{\text {EC }}$ (Supplementary Fig. 5d). For CCL2, a

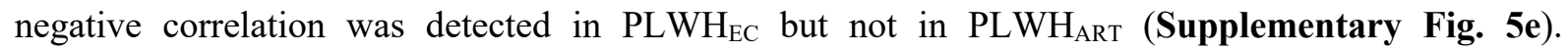
Collectively, this data indicates that although PLWH $_{\mathrm{ART}}$ have higher proportion of infected cells compared to $\mathrm{PLWH}_{\mathrm{EC}}$, a larger fraction of the integrated HIV-1 carries hypermutations or 3'deletions within the viral genome. Furthermore, the frequency of intact provirus out of total HIV-1 DNA is low in both PLWHEC and PLWH $\mathrm{PRT}_{\mathrm{ART}}$

Altered metabolic pathways in $\mathrm{CD4}^{+} \mathrm{CCR6}^{+}$cells are characterised by an upregulation in p53 signalling and apoptosis in $\mathrm{PLWH}_{\mathrm{EC}}$

211 The subpopulation of $\mathrm{CD} 4^{+} \mathrm{CCR} 6^{+}$cells has been described as more permissive towards $\mathrm{HIV}-1$ and 212 targets for productive infection ${ }^{31,32}$. To further characterise the $\mathrm{CD} 4^{+} \mathrm{T}$ cells that express or lack the 213 CCR6 receptor we sorted out these cell populations using fluorescence activated cell sorting (FACS) 214 from $\mathrm{HC}(n=3), \mathrm{PLWH}_{\mathrm{EC}}(n=6)$ and $\mathrm{PLWH}_{\mathrm{ART}}(n=6)$ (Fig. 5a). The PLWH $\mathrm{ART}_{\mathrm{A} T}$ samples were selected from PLWH on long-term suppressive ART (median 20 years, IQR; 17.5-22.25). First, as the HIV-1

216 reservoir has been proposed to reside in $\mathrm{CD} 4^{+} \mathrm{CCR} 6^{+} \mathrm{T}$ cells, we measured the amount of integrated 217 HIV-1 in PLWHEC $(n=5)$ and PLWH PRT $(n=3)$. However, the variation within the data and the small 218 sample size made the comparison inconclusive (Supplementary Fig. 6a). To evaluate the specific 219 proteomic profile of $\mathrm{CD} 4^{+} \mathrm{CCR} 6^{-}$and $\mathrm{CD} 4^{+} \mathrm{CCR} 6^{+}$cell populations we isolated these cell populations 
by FACS (15,000-30,000 cells) and performed mass spectrometry-based proteomics using a quantitative tandem mass tags (TMT) labelling approach. Due to low protein detection by mass spectrometry four $\mathrm{PLWH}_{\mathrm{EC}}$ samples, two samples out of each of the $\mathrm{CCR}^{+}$and $\mathrm{CCR} 6^{-}$cell populations were excluded from the analysis. The principal component analysis (PCA) identified heterogeneity among the samples (Supplementary Fig. 6b). Differential expression analysis was unable to find statistically significant regulated proteins due to heterogeneity among samples and low sample size. Therefore, functional analysis was performed using a consensus scoring approach based on multiple protein set analysis (PSA) run by incorporating the directionality of protein abundance. Using the group specific consensus scores $\left(\mathrm{PLWH}_{\mathrm{EC}}\right.$ vs PLWH $\left.\mathrm{ART}_{\mathrm{A}}\right)$ and directionality classes, we identified distinct upregulation of oxidative phosphorylation $(\mathrm{OXPHOS})(\operatorname{adj} \mathrm{p}<0.05)$ and interferon- $\alpha$ response $(\operatorname{adj} \mathrm{p}<0.05)$ and distinct downregulation of glycolysis (adj $\mathrm{p}<0.05$ ) in both $\mathrm{CD}^{+} \mathrm{CCR}^{+}$and $\mathrm{CD}^{+} \mathrm{CCR} 6^{-}$cells while using the MSigDB hallmark gene-set (Fig. 5b). Similarly, the KEGG gene-set also identified a distinct upregulation of OXPHOS (adj $\mathrm{p}<0.1$ ), and a distinct downregulation of other pathways of amino acid and carbohydrate metabolism (Supplementary Fig. 6c). This data indicates that the metabolic trade-off of increased OXPHOS and reduced glycolysis is elevated in PLWHEC compared to PLWH $\mathrm{ART}_{\mathrm{ART}}$ (Fig. 5c). The relative abundance of the OXPHOS proteins were higher in $\mathrm{PLWH}_{\mathrm{EC}}$ compared to HC while lower

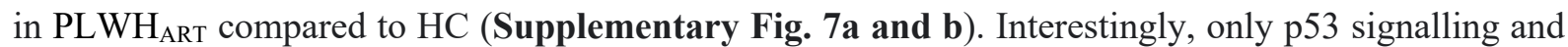
apoptosis were distinctly upregulated in $\mathrm{CD}^{+} \mathrm{CCR}^{+}(\operatorname{adj} \mathrm{p}<0.05)$ (Fig. 5b) and both pathways had unique features in $\mathrm{PLWH}_{\mathrm{ART}}$ (Fig. 5d and e). The majority of the PLWH $\mathrm{ART}_{\mathrm{A} T}$ clustered together. There were two downregulated proteins in PLWHEC (ISCU and LDHB) that were common between OXPHOS and p53 signalling. Furthermore, four proteins (CASP7, VDAC2, RHOT2 and GPX4) were common between OXPHOS and apoptosis, while seven proteins (FAS, TAP1, FDXR, TXNIP, CASP1, APP and HMOX1) were common between p53 signalling and apoptosis among the proteins upregulated in $\mathrm{PLWH}_{\mathrm{EC}}$ compared to $\mathrm{PLWH}_{\mathrm{ART}}$ (Fig. 5f). Taken together, this data shows how the $\mathrm{CD}^{+} \mathrm{CCR} 6^{+}$cell population have a unique profile in PLWHEC. The co-regulation of OXPHOS, p53-signalling and apoptosis may be a contributing factor to control of HIV-1 infection.

\section{Discussion}

248 In our study, a unique cell type expression profile of CCR2 and CCR6 was identified in PLWHEC, while $249 \mathrm{PLWH}_{\mathrm{ART}}$ exhibited an expression profile more like HC. CCR6 ${ }^{+}$cells were thus decreased on both CD4 ${ }^{+}$ and $\mathrm{CD}^{+} \mathrm{T}$ cells in $\mathrm{PLWH}_{\mathrm{EC}}$ but not in PLWH $\mathrm{ART}_{\text {T }}$. Furthermore, the $\mathrm{CD} 4^{+} \mathrm{T}$ cells exhibited a protein profile of modulated metabolic activity towards OXPHOS and reduced protein levels involved in glycolysis in $\mathrm{PLWH}_{\mathrm{EC}}$, irrespective of CCR6 status. Collectively, as $\mathrm{CD}^{+} \mathrm{CCR}^{+}$cells have been reported to be highly permissive to HIV-1 ${ }^{31,32}$, and substantial contributors to the HIV-1 reservoir, low levels of $\mathrm{CD}^{+} \mathrm{CCR}^{+}$cells, metabolic modulation, and enrichment of proteins involved in cell death, could potentially contribute to natural control of HIV-1. 
Within the $\mathrm{CD}^{+}{ }^{+} \mathrm{T}$ cell subpopulation, CCR6 expression is a marker of some memory $\mathrm{T}$ cells subsets, namely Th17 cells, NKT cells, and $\gamma \delta \mathrm{T}$ cells ${ }^{33}$. Of these cell populations, the Th17 cells promote a strong pro-inflammatory response and are drivers of chronic inflammation and autoimmune diseases ${ }^{34}$ ${ }^{35}$. Earlier studies have shown how memory $\mathrm{CD} 4^{+} \mathrm{CCR} 6^{+} \mathrm{T}$ cells express a Th17 lineage profile and high susceptibility towards HIV-1 ${ }^{36}$. In our cohort, the proportion of $\mathrm{CCR}^{+}$cells were reduced in PLWHEC compared to both PLWH $\mathrm{ART}_{\text {and }}$ HC. CCR6 is also a homing marker for gut associated lymphoid tissue $(\mathrm{GALT}){ }^{37}$. Therefore, the causative factor of reduced $\mathrm{CD} 4^{+} \mathrm{CCR} 6^{+}$cells in peripheral blood in $\mathrm{PLWH}$

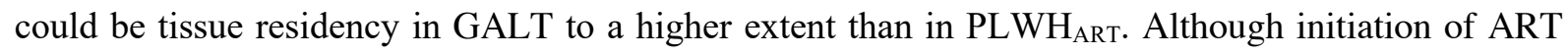
restores the intestinal $\mathrm{CD}^{+} \mathrm{T}$ cell compartments, the severe depletion induced during the early stages of infection cannot be fully recovered ${ }^{38}$, which contributes to exacerbation of HIV-1 through microbial translocation and hyperactivation of the immune system ${ }^{39} 40$. Collectively, an increased gut homing potential of CCR6 ${ }^{+}$cells in $\mathrm{PLWH}_{\mathrm{EC}}$, could help to restore the imbalance of $\mathrm{CD} 4^{+} \mathrm{T}$ cells in GALT and reduce systemic chronic inflammation among these patients.

We have earlier showen that low expression of CCR6 and high levels of its ligand, CCL20, may result in low levels of inflammation in $\mathrm{PLWH}_{\mathrm{EC}}{ }^{25}$. Herein, we detected increased plasma levels of CCL20 in PLWH, independent of HIV-1 status compared to HC, confirming earlier observations ${ }^{42}$. CCL20 has previously been described to have an effect on the infection step of HIV-1, migration of immune cells and immune cell function. However, we did not see any difference in CCL20 levels between PLWHEC and PLWH $\mathrm{PRT}_{\text {ART }}$ Therefore, most likely it is the $\mathrm{CD}^{+} \mathrm{CCR}^{+}$cells, rather than levels of CCL20, that in $\mathrm{PLWH}_{\mathrm{EC}}$ could contribute to natural control of HIV-1.

Furthermore, $\mathrm{CD}^{+} \mathrm{CCR} 6^{+}$and $\mathrm{CD} 8^{+} \mathrm{CCR} 6^{+}$cells decline in untreated patients with HIV $-1{ }^{43}$ since the $\mathrm{CCR}^{+}$cells are more susceptible towards apoptosis and therefore involved in the depletion of memory $\mathrm{T}$ cells. However, we did not detect any alterations in $\mathrm{CD} 4^{+} \mathrm{CCR} 6^{+}$compartments in $\mathrm{PLWH}_{\mathrm{ART}}$ compared to $\mathrm{HC}$, although for $\mathrm{CD} 8^{+} \mathrm{CCR} 6^{+}$cells there was a reduction in both patient-groups. $\mathrm{We}$ identified the $\mathrm{CD}^{+} \mathrm{CCR}^{+}$cells as having increased proteins involved in apoptosis and p53 signalling in $\mathrm{PLWH}_{\mathrm{EC}}$, indicating an increased sensitivity towards apoptosis, possibly as a result of HIV-1 proteins, activation-induced cell death or induced expression of death receptors, e.g., FasL and TRAIL ${ }^{44}$. Since these cells are not restored in the GALT during ART, it can be postulated that the $\mathrm{CD} 4{ }^{+} \mathrm{CCR} 6^{+}$cell population is a major target for HIV-1 infection that in PLWH contributes to the inflammatory environment in both GALT and peripheral blood. In PLWHEC, these cells may therefore be more susceptible towards apoptosis, possibly through expression of pro-apoptotic HIV-1 proteins during infection or reactivation from latency. Furthermore, p53 is a tumour suppressor gene that during HIV-1 infection is activated by interferon $\alpha$ and $\beta$ stimulation of immune cells ${ }^{45}$. Activity of p53 can interfere with HIV-1 replication by inhibiting reverse transcription of the virus and suppressing the activity of the transactivator tat, needed for viral transcription ${ }^{46}$. Therefore, an enrichment of p53 signalling in 
$291 \mathrm{PLWH}_{\mathrm{EC}}$ could contribute to natural control of HIV-1 infection in $\mathrm{CD} 4^{+} \mathrm{CCR} 6^{+} \mathrm{T}$ cells by reducing 292 replication of HIV-1 and inducing apoptosis upon latency reversal. Additionally, $\mathrm{PLWH}_{\mathrm{EC}}$ exhibited an 293 enrichment of proteins involved in OXPHOS together with decreased number of proteins involved in 294 glycolysis, irrespective of CCR6 expression. Increased glycolysis is a hallmark for T cell activation but 295 also upregulated during HIV-1 infection for cells to sustain the energy demanding process of virus 296 production ${ }^{47} 48$. Generally, activation of a T cell induces a shift from OXPHOS to aerobic glycolysis in 297 a similar fashion as the metabolic reprogramming occurring during activation from latency ${ }^{49,50}$. 298 Therefore, the metabolic state of the $\mathrm{CD}^{+} \mathrm{T}$ cell compartment in PLWH $\mathrm{EC}_{\mathrm{EC}}$, independently of CCR6 299 expression indicates a unique proportion of CD4 T cell subsets and metabolic reprogramming.

300 Persistent HIV-1 is a driver of chronic immune activation and inflammation. In our cohort, PLWHEC 301 had a reduced amount of integrated HIV-1 in total PBMCs compared to PLWHART, correlating to earlier 302 observations ${ }^{23}$. On the other hand, treatment duration did not significantly alter the proportion of latently 303 infected cells probably due to the stability of the reservoir during long-term ART. During suppressive 304 therapy, persisting HIV-1 contributes to low level of viral replication and cell to cell spread driving the 305 chronic immune cell activation and inflammaging that ultimately sustains the HIV-1 reservoir in the body, together with homeostatic proliferation ${ }^{51} 52$. However, evaluation of the intact provirus by IPDA showed a decrease of 3'deletion/hypermutation in the provirus from PLWHEC compared to PLWH ART. $_{\text {. }}$ Earlier studies have shown that 3 ' deletion clones decline during suppressive therapy ${ }^{53}$. These specific mutated proviruses may be under negative selection due to their capacity to stimulate the immune system ${ }^{53} 54$. With a reduced proportion of provirus in $\mathrm{PLWH}_{\mathrm{EC}}$ compared to $\mathrm{PLWH}_{\mathrm{ART}}$, it could be hypothesised that this negative selection is stronger in $\mathrm{PLWH}_{\mathrm{EC}}$, explaining the lower proportion of integrated HIV1.

313 Monocytes produce pro-inflammatory cytokines where IM are efficient producers of IL6 and IL8 in 314 response to microbial pathogens together with CM, whereas NCM produce TNF $\alpha$, CCL3 and IL-1 $\beta$ in 315 response to viral elements ${ }^{55}$. In our cohort, elevated levels of IM were detected in PLWH $\mathrm{EC}_{\mathrm{EC}}$, indicative 316 of an increased anti-inflammatory response. However, the expression profile of CCR2 on IM was similar

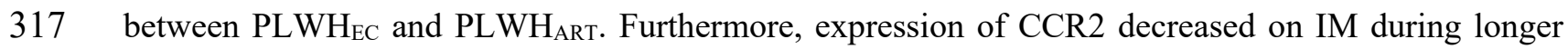
318 suppressive therapy in our longitudinal $\mathrm{PLWH}_{\mathrm{ART}}$ cohort and a similar trend was seen in PLWHEC and 319 PLWH $_{\text {ART }}$ compared to HC. Monocytes are targets of HIV-1 infection and drivers of inflammation and 320 comorbidities during HIV-1 infection ${ }^{56}$. Therefore, the expansion of IM could indicate a role in a 321 heightened pathogen defence in $\mathrm{PLWH}_{\mathrm{EC}}$.

322 Overall, our study showed that the chemokine receptor profile in $\mathrm{PLWH}_{\mathrm{ART}}$ is more similar towards $\mathrm{HC}$ 323 on lymphocytic cell populations, and $\mathrm{PLWH}_{\mathrm{EC}}$ on monocytic cell populations. $\mathrm{PLWH}_{\mathrm{EC}}$ have a reduced

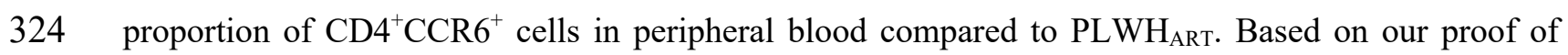
principle proteomic analysis we hypothesise that the $\mathrm{CD} 4^{+} \mathrm{CCR} 6^{+}$cells are more susceptible to 
apoptosis, while $\mathrm{CD}^{+} \mathrm{T}$ cells in $\mathrm{PLWH}_{\mathrm{EC}}\left(\mathrm{CCR} 6^{-} / \mathrm{CCR} 6^{+}\right)$exhibit decreased metabolic activation, indicative of a reduced activation state. Collectively, these lymphocytic cell populations may aid in facilitating the unique characteristics of $\mathrm{PLWH}_{\mathrm{EC}}$ and contribute to chronic inflammation and $\mathrm{HIV}-1$ persistence during suppressive therapy.

\section{$331 \quad$ Methods}

\section{Patient material}

333 The clinical cohort consisted of $\mathrm{HIV}-1^{+}$individuals on suppressive therapy ( $\mathrm{PLWH}$ ART, $n=54$ ), elite controllers ( $\mathrm{PLWH}_{\mathrm{EC}}, n=14$ ) collected from the InfCareHIV cohort at the Department of Infectious Diseases, Karolinska University Hospital, Sweden, and HIV-1 negative individuals (HC, $n=18$ ). The cohort was age matched and the median duration of treatment was eight years (IQR; 6.75-19). Cohort characteristics can be viewed in Table 1 and treatment regimen for PLWHART in Supplementary Table 1. The cohort also consisted of longitudinal data within the PLWH $\mathrm{ART}_{\text {Troup }}(\mathrm{T} 1, n=10$, and T2, $n=10$ ) (Supplementary Table 2). Patient material consisted of plasma and PBMCs collected from whole blood using Ficoll-Paque (Cytiva).

This study was approved by the Regional Ethics Committee of Stockholm (Dnr 2013/1944-31/4) and amendments (2014/920-32 and 2019-05585) together with (Dnr 2009/1485-31) and amendments (2015/1765-32 and 2019-05584). Informed consent was given from all participants prior inclusion and data was anonymized and delinked before analysis.

\section{Flow cytometry and FACS}

348 PBMCs were stained for cell surface markers for $20 \mathrm{~min}$ at room temperature (RT) using $5 \mu \mathrm{L} / 10^{6}$ 349 PBMCs of CD3 (OKT3, FITC, Biolegend \#317306), CD4 (SK3, BUV395, BDbioscience \#563550), 350 CD8 (RPA-T8, APC, Biolegend \#301014), CD14 (M5E2, BV510, Biolegend \#301842), CD16 (3G8 , 351 BV786, BDbioscience \#563690), CCR2 (1D9, BB700, BDbioscience \#747847), CCR3 (5E8, BV421, 352 Biolegend \#310714), CCR5 (2D7, PE-CF594, Bdbioscience \#562456), CCR6 (G034E3, BV711, 353 Biolegend \#353436), and Near IR viability dye (Invitrogen \#L10119). Cells were subsequently washed 354 two times using FACS buffer (PBS with 2\% FBS and 2 mM EDTA) and fixed 15 min at RT using 2\% 355 paraformaldehyde. Results were acquired on BD FACS Symphony (BD Bioscience, USA) using laser 356 and filer settings as indicated for BUV395, BV421, BV510, BV711, BV786, FITC, BB700, PE-CF594, 357 APC and Near IR respectively; 355nm UV (100mW) 379/28, 405nm violet (100mW), 450/50, 525/50 358 (505 LP), 710/50 (685 LP), 810/40 (770 LP), 488nm blue (200mW) 530/30 (505 LP), 710/50 (685 LP), $561 \mathrm{~nm} \mathrm{Y/G} \mathrm{(200mW),} \mathrm{610/20} \mathrm{(600LP)} \mathrm{and} \mathrm{637nm} \mathrm{Red} \mathrm{(140mW),} \mathrm{670/30} \mathrm{and} \mathrm{780/60} \mathrm{(750LP).} \mathrm{Cell}$ expression analysis and t-SNE was performed using FlowJo 10.6 (Tree Star Inc). Co-expression analysis was performed using the Boolean gating function and the visualisation of complex data using Spice ${ }^{57}$. 
362 Data from flow cytometry detection in $\operatorname{PLWH}_{\mathrm{EC}}(n=14)$ and $\mathrm{HC}(n=8)$ samples were used from our 363 earlier publication ${ }^{25}$.

365 Cryopreserved PBMCs were used for fluorescent activated cell sorting (FACS) for subsequent 366 proteomic analysis and DNA extraction. Cells were thawed and stained using anti-human CD3 (OKT3, 367 FITC, Biolegend \#317306), CD4 (SK3, PE-Cy5, Biolegend \# 344654), CD8 (RPA-T8, BV570, 368 Biolegend \#301038), CD14 (M5E2, BV510, Biolegend \#301842), CD16 (3G8, BV786, BDbioscience 369 \#563690), CCR6 (G034E3, BV711, Biolegend \#353436) for $30 \mathrm{~min}$ at $4^{\circ} \mathrm{C}$ and subsequent FVS450 370 viability dye (BDbioscience \#562247) for $10 \mathrm{~min}$ at $\mathrm{RT}$. $\mathrm{CD} 4^{+} \mathrm{CCR} 6^{-}$and $\mathrm{CD} 4^{+} \mathrm{CCR} 6^{+}$cell were 371 collected for downstream analysis. Cells were sorted using SONY MA900 (SONY cooperation, Tokyo, 372 Japan).

\section{DNA extraction}

375 Total DNA was extracted from whole PBMCs or collected cell populations by QIAamp Mini or QIAamp 376 Micro DNA isolation kit (Qiagen), according to the manufacturers protocol.

\section{HIV-1 DNA quantification}

379 Patient PBMCs or sorted cells were used for quantification of total HIV-1 DNA by Internally Controlled 380 qPCR (IC-qPCR) developed by Vicenti et al. $2018^{58}$. In brief, total HIV-1 DNA was quantified using 381 Takara Universal mastermix on the $\mathrm{ABI} 7500 \mathrm{~F}$ and normalized to $\beta$-Actin levels, primer and probe 382 details are described in Supplementary Table 3. For each sample, 200-500 ng were run per reaction in 383 duplicates and data was normalised to background detection in HC.

\section{Digital droplet PCR and Intact proviral DNA assay (IPDA)}

386 Digital droplet PCR (ddPCR) was performed using the QX200 Droplet Digital qPCR System (RioRad). 387 Samples consisting of $10 \mu \mathrm{L}$ Supermix for probes (no dUTPs) (Bio-Rad), $900 \mathrm{nM}$ primers, $250 \mathrm{nM}$ 388 probe (labeled with either HEX or FAM) or $500 \mathrm{ng}$ of genomic sample DNA and $50 \mathrm{ng}$ of DNA for 389 RPP30 quantification for shearing analysis and normalization, were emulsified with the QX200 droplet 390 generator. PCR reactions were subsequently performed on a C1000 thermal cycler (Bio-Rad) using the 391 following thermal cycling protocol: $10 \mathrm{~min}$ at $95{ }^{\circ} \mathrm{C}$ for enzyme activation followed by 45 annealing / 392 extension cycles of $30 \mathrm{~s}$ at $94{ }^{\circ} \mathrm{C}$ and $60 \mathrm{~s}$ at $57{ }^{\circ} \mathrm{C}$ and a final enzyme deactivation step at $98{ }^{\circ} \mathrm{C}$ for 10 393 min. Subsequently, samples were read with the QX200 Droplet Reader (Bio-Rad) and analyzed with the 394 QuantaSoft software version 1.5 (Bio-Rad).

395 For the quantification of intact, 5' deleted, and 3' deleted / hypermutated proviruses, the Intact Proviral 396 DNA Assay was performed as previously described ${ }^{28}$. Briefly, a first ddPCR was performed as 397 described above with primer/probe combinations for the packaging signal ( $\Psi)$, which frequently shows 398 small mutations. The second reaction targets the HIV-1 Rev response element, present in the Env coding 
region. Two hydrolysis probes were used in this reaction: a HEX-labeled probe specific for the intact

400 proviral sequence and an unlabeled probe specific for the APOBEC-3G hypermutation, which competes

401 with the HEX-labeled probe and results in no signal in the case of APOBEC hypermutations. Duplicate

402 wells for IPDA were merged during analysis. All primer and probe details can be viewed in

\section{Supplementary Table 3.}

404

\section{Targeted Proteomic profiling}

406 Plasma levels of CCL20 was measured using Human Quantikine ELISA kits (R\&Dsystems) according 407 to the manufacturers protocol. Data for plasma CCL2 was used from our earlier publication ${ }^{18}$. In brief, 408 plasma levels were analysed using proximity extension assay with the OLINK $®$ immune-oncology 409 panel (OLINK). Proteins are reported as normalised protein expression levels (NPX) where Ct values 410 are normalised by the subtraction of the extension control and inter-plate control.

\section{Mass Spectrometry based proteomics}

413 Proteins were extracted by adding $8 \mathrm{M}$ urea in $100 \mathrm{mM}$ ammonium bicarbonate lysis buffer. Samples 414 were vortexed and sonicated in cold bath for $10 \mathrm{~min}$. Protein concentration was estimated with Pierce 415 Micro BCA assay (ThermoFisher Scientific). Extracted proteins were reduced with $10 \mathrm{mM}$ dithiothreitol 416 for $60 \mathrm{~min}$ at $37^{\circ} \mathrm{C}$ and alkylated with $40 \mathrm{mM}$ chloroacetamide (CAM) for $30 \mathrm{~min}$ at room temperature. 417 Proteins were digested with trypsin (sequencing grade from Promega) with a 1:20 ratio enzyme:protein 418 overnight at $37^{\circ} \mathrm{C}$. Resulting peptides were cleaned-up using C-18 stage tips (ThermoFisher Scientific) 419 according to manufacturer's instructions and dried in a vacuum concentrator (Eppendorf). Cleaned 420 peptides were labeled with TMT11plex ${ }^{\mathrm{TM}}$ tags (ThermoFisher Scientific) following manufacturer's 421 instructions. Briefly, peptides were resuspended in $100 \mathrm{mM}$ triethylammonium bicarbonate and 422 individual TMT tags were dissolved in dry acetonitrile (ACN). Peptides were labeled by adding the 423 respective TMT tag solution, the mix was incubated for 1 hour at room temperature, the reaction was 424 quenched by adding 5\% hydroxylamine and incubated for $15 \mathrm{~min}$ at RT. Labeled peptides were mixed 425 in three different batches according to scheme described in Supplementary Table 4, a pool of samples 426 was used across the three batches to normalize and evaluate batch effect (TMT channel 131C).

Labeled peptides were fractionated using Pierce High $\mathrm{pH}$ Reversed-Phase Peptide Fractionation Kit columns (ThermoFisher Scientific) following manufacturer's instructions. Briefly, columns were 430 washed with $100 \% \mathrm{ACN}$ and then conditioned with $0.1 \%$ trifluoroacetic acid (TFA). Peptides were 431 dissolved in $0.1 \%$ TFA and loaded into the column by centrifugation at $3000 \mathrm{x} g$ for 2 min. Columns 432 were washed with $5 \% \mathrm{ACN}$ in $0.1 \%$ triethanolamine (TEA), and peptides were eluted in eight different 433 fractions by centrifugation at $3000 \mathrm{x} g$ according to the ACN concentration in $0.1 \%$ TEA solutions: 10 , $43412.5,15,17.5,20,22,5,25$ and $50 \%$. All fractions were dried down in a vacuum concentrator (Eppendorf) prior to LC-MS/MS analysis. 
437 Fractionated peptides were analyzed on an Ultimate 3000 UHPLC (ThermoFisher Scientific) 438 hyphenated to an Orbitrap ${ }^{\mathrm{TM}}$ Lumos $^{\mathrm{TM}}$ mass spectrometer (ThermoFisher Scientific). Peptides were 439 loaded in an Acclaim PepMap trap column, $2 \mu \mathrm{m}$ x $75 \mu \mathrm{m}$ ID x 2 cm (Thermo Scientific) and separated 440 in an EASY-Spray ${ }^{\mathrm{TM}}$ HPLC Column, $2 \mu \mathrm{m}$ x $75 \mu \mathrm{m}$ ID x $500 \mathrm{~mm}$ (Thermo Scientific) using a $120 \mathrm{~min}$ 441 linear gradient. Data was acquired in data dependent acquisition (DDA) mode, isolating the top 20 most intense precursors at 120,000 mass resolution in the mass range of $\mathrm{m} / \mathrm{z} 375-1400$, maximum injection time (IT) of $50 \mathrm{~ms}$ and dynamic exclusion of $30 \mathrm{~s}$, precursors were isolated with $0.7 \mathrm{Th}$ width. MS/MS scans were obtained using high collision energy of $34 \%$, resolution of 45,000 and maximum IT of 86 ms.

446 Proteins were searched against both SwissProt human and HIV-1 databases using the search engine 447 Mascot Server v2.5.1 (MatrixScience Ltd) in Proteome Discoverer v2.5 (ThermoFisher Scientific) 448 software environment allowing maximum two missed cleavages. Oxidation of methionine, deamidation 449 of asparagine and glutamine, TMT6plex modification of lysine and N-termini were set as variable modifications, while carbamidomethylation of cysteine was used as fixed modification. The false discovery rate (FDR) was set to $1 \%$.

\section{Bioinformatics analysis}

454 The data was normalized using quantile method from the R/Bioconductor package NormalyzerDE 455 v1.4.0. Missing data was imputed by employing nearest neighbour averaging method using impute.knn 456 function from R package impute v1.60.0. Default setting was used for the data imputation. Technical 457 variations in the data due to batch effect was removed using $\mathrm{R}$ function Combat from the package sva 458 v3.34.0. Differential expressions analysis was performed with R/Bioconductor package Limma v3.42.2. 459 Gene set enrichment analysis was performed using R/Bioconductor package piano v2.2.0. Gene level t460 statistics given by Limma, and hallmark gene set downloaded from MSigDB were used to find 461 significantly enriched gene-sets. Gene-sets with Benjamini-Hochberg adjusted p-values less than 0.2 462 were considered as significantly enriched. Volcano and bubble plots were created using R package 463 ggplot2 v3.3.2.

\section{Statistical analysis}

466 Statistical analysis was performed using two-tailed Mann-Whitney U-test, and Wilcoxon ranked sum test or paired t-test for longitudinal data, based on the distribution of the data in Prism v8 (GraphPad 468 Prism Software). Statistical significance was $p<0.05$ for all analysis. Data was visualized using the 469 package ggplot2 v.3.3.2 in Rstudio (v.1.3.1056). Correlation analysis was performed using Spearman's correlation $(p<0.05)$ in Rstudio (v.1.3.1056). 
The mass spectrometry proteomics data have been deposited to the ProteomeXchange Consortium via the PRIDE partner repository with the dataset identifier PXD027749.

\section{Acknowledgements}

477 This study was founded by the Swedish Research Council Grants (2017-01330 to U.N. and 2017-05848 to A.S.) and Karolinska Institutet Stiftelser och Fonder. UN was also supported by the Swedish Research Council Interdisciplinary Grant (2018-06156). We would like to thank Assisstant professor Robert van Domselaar for providing intellectual imput on immunological aspects of the manuscript. Furthermore, we would like to acknowledge Dr. Sivasankaran Munusamy Ponnan for performing the t-SNE plots.

\section{Author contribution}

484 U.N. conceived the study; A.S. initiated, designed, and governed the Swedish Elite controller cohort; U.N. and S.S.A. planned the experiments; S.S.A., S.K., S.G., B.J., and M.S. performed the research; J.E.R., and A.V. performed the proteomic analysis; A.T.A. performed the bioinformatics analysis. A.S., P.N., and J.V contributed with patient material. S.S.A. and U.N. analysed the data. S.G. and P.S. contributed with intellectual input. S.S.A. and U.N. wrote the manuscript. All authors critically reviewed and revised the manuscript.

490

\section{Competing interests}

492 The authors declare no competing interest.

\section{References}

1. Cohn LB, Chomont N, Deeks SG. The Biology of the HIV-1 Latent Reservoir

2. Khanal S, Schank M, El Gazzar M, Moorman JP, Yao ZQ. HIV-1 Latency and Viral and Implications for Cure Strategies. Cell Host \& Microbe (2020). Pathways Involved in HIV Latency Studies. Cells 10, (2021).

3. Chomont $\mathrm{N}$, et al. HIV reservoir size and persistence are driven by $\mathrm{T}$ cell survival and homeostatic proliferation. Nat Med 15, 893-900 (2009).

4. Pedro KD, Henderson AJ, Agosto LM. Mechanisms of HIV-1 cell-to-cell transmission and the establishment of the latent reservoir. Virus Res 265, 115-121 (2019).

5. Massanella M, Fromentin R, Chomont N. Residual inflammation and viral reservoirs: alliance against an HIV cure. Curr Opin HIV AIDS 11, 234-241 (2016).

6. Choe $\mathrm{H}$, et al. The $\beta$-Chemokine Receptors CCR3 and CCR5 Facilitate Infection by Primary HIV-1 Isolates. Cell 85, 1135-1148 (1996). 
7. Alkhatib G, Berger EA, Murphy PM, Pease JE. Determinants of HIV-1 coreceptor

8. He J, et al. CCR3 and CCR5 are co-receptors for HIV-1 infection of microglia. Nature 385, 645-649 (1997).

9. Anderson JL, et al. Human Immunodeficiency Virus (HIV)-Infected CCR6+ Rectal CD4+ $T$ Cells and HIV Persistence On Antiretroviral Therapy. The Journal of Infectious Diseases 221, 744-755 (2020).

10. Gosselin A, et al. HIV persists in CCR6+CD4+ T cells from colon and blood during antiretroviral therapy. AIDS (London, England) 31, 35-48 (2017).

11. Packard TA, Herzig E, Roan NR, Greene WC. Establishing the HIV reservoir: HIVsusceptible cells and the signals that recruit them. The Journal of Immunology 198, 125.121 (2017).

12. Wang Z, Shang H, Jiang Y. Chemokines and Chemokine Receptors: Accomplices for Human Immunodeficiency Virus Infection and Latency. Frontiers in immunology 8, 1274-1274 (2017).

13. Zicari S, et al. Immune Activation, Inflammation, and Non-AIDS Co-Morbidities in HIVInfected Patients under Long-Term ART. Viruses 11, (2019).

14. Nakayama K, et al. Imbalanced Production of Cytokines by T Cells Associates with the Activation/Exhaustion Status of Memory T Cells in Chronic HIV Type 1 Infection. AIDS research and human retroviruses $28,702-714$ (2011).

15. Babu H, et al. Systemic Inflammation and the Increased Risk of Inflamm-Aging and AgeAssociated Diseases in People Living With HIV on Long Term Suppressive Antiretroviral Therapy. Frontiers in immunology 10, (2019).

16. Babu $\mathrm{H}$, et al. Plasma Metabolic Signature and Abnormalities in HIV-Infected Individuals on Long-Term Successful Antiretroviral Therapy. Metabolites 9, 210 (2019).

17. Sperk $M$, Zhang $W$, Nowak P, Neogi U. Plasma soluble factor following two decades prolonged suppressive antiretroviral therapy in HIV-1-positive males: A cross-sectional study. Medicine (Baltimore) 97, e9759-e9759 (2018).

18. Zhang W, et al. Transcriptomics and Targeted Proteomics Analysis to Gain Insights Into the Immune-control Mechanisms of HIV-1 Infected Elite Controllers. EBioMedicine 27, 40-50 (2018).

19. Olson $A D$, et al. An evaluation of HIV elite controller definitions within a large seroconverter cohort collaboration. PloS one 9, e86719-e86719 (2014). 
20. Poropatich K, Sullivan DJ. Human immunodeficiency virus type 1 long-term nonprogressors: the viral, genetic and immunological basis for disease non-progression. Journal of General Virology 92, 247-268 (2011).

21. International HIVCS, et al. The major genetic determinants of HIV-1 control affect HLA class I peptide presentation. Science (New York, NY) 330, 1551-1557 (2010).

22. McLaren PJ, et al. Fine-mapping classical HLA variation associated with durable host

23. Jiang $\mathrm{C}$, et al. Distinct viral reservoirs in individuals with spontaneous control of HIV-1. Nature 585, 261-267 (2020).

24. Hocini $\mathrm{H}$, et al. HIV Controllers Have Low Inflammation Associated with a Strong HIVSpecific Immune Response in Blood. Journal of Virology 93, e01690-01618 (2019).

25. Sperk $M$, et al. Distinct lipid profile, low-level inflammation and increased antioxidant defense signature in HIV-1 elite control status. iScience, 102111 (2021).

26. Anzinger JJ, Butterfield TR, Angelovich TA, Crowe SM, Palmer CS. Monocytes as regulators of inflammation and HIV-related comorbidities during CART. Journal of immunology research 2014, 569819-569819 (2014).

27. Jaworowski A, Hearps AC, Angelovich TA, Hoy JF. How Monocytes Contribute to Increased Risk of Atherosclerosis in Virologically-Suppressed HIV-Positive Individuals Receiving Combination Antiretroviral Therapy. Frontiers in immunology 10, 1378-1378 (2019).

28. Bruner KM, et al. A quantitative approach for measuring the reservoir of latent HIV-1 proviruses. Nature 566, 120-125 (2019).

29. Cameron PU, et al. Establishment of HIV-1 latency in resting CD4+ T cells depends on chemokine-induced changes in the actin cytoskeleton. Proceedings of the National Academy of Sciences of the United States of America 107, 16934-16939 (2010).

30. Ghosh M, Shen Z, Schaefer TM, Fahey JV, Gupta P, Wira CR. CCL20/MIP3alpha is a novel anti-HIV-1 molecule of the human female reproductive tract. American journal of reproductive immunology (New York, NY : 1989) 62, 60-71 (2009).

31. Gosselin A, et al. Peripheral blood CCR4+CCR6+ and CXCR3+CCR6+CD4+ T cells are highly permissive to HIV-1 infection. Journal of immunology (Baltimore, Md : 1950) 184, 1604-1616 (2010).

32. Planas $D$, et al. HIV-1 selectively targets gut-homing CCR6+CD4+ T cells via mTORdependent mechanisms. JCI Insight 2, (2017). 
33. Lee AYS, Korner H. CCR6/CCL20 chemokine axis in human immunodeficiency virus immunity and pathogenesis. The Journal of general virology 98, 338-344 (2017).

34. Yasuda K, Takeuchi Y, Hirota K. The pathogenicity of Th17 cells in autoimmune diseases. Seminars in Immunopathology 41, 283-297 (2019).

35. Korn T, Bettelli E, Oukka M, Kuchroo VK. IL-17 and Th17 Cells. Annual Review of Immunology 27, 485-517 (2009).

36. Monteiro $P$, et al. Memory CCR6+CD4+T Cells Are Preferential Targets for Productive HIV Type 1 Infection Regardless of Their Expression of Integrin $\beta 7$. The Journal of Immunology 186, 4618 (2011).

37. Wang C, Kang SG, Lee J, Sun Z, Kim CH. The roles of CCR6 in migration of Th17 cells and regulation of effector T-cell balance in the gut. Mucosal Immunology 2, 173-183 (2009).

38. Guadalupe $M$, et al. Severe CD4+ T-Cell Depletion in Gut Lymphoid Tissue during Primary Human Immunodeficiency Virus Type 1 Infection and Substantial Delay in Restoration following Highly Active Antiretroviral Therapy. Journal of Virology 77, 11708-11717 (2003).

39. Sandler NG, Douek DC. Microbial translocation in HIV infection: causes, consequences and treatment opportunities. Nature Reviews Microbiology 10, 655-666 (2012).

40. Chun T-W, et al. Persistence of HIV in Gut-Associated Lymphoid Tissue despite LongTerm Antiretroviral Therapy. The Journal of Infectious Diseases 197, 714-720 (2008).

41. Valverde-Villegas JM, Matte MCC, Medeiros RMd, Chies JAB. New Insights about Treg and Th17 Cells in HIV Infection and Disease Progression. Journal of Immunology Research 2015, 647916 (2015).

42. Fontaine J, Poudrier J, Roger M. Short Communication: Persistence of High Blood Levels of the Chemokines CCL2, CCL19, and CCL20 During the Course of HIV Infection. AIDS research and human retroviruses 27, 655-657 (2010).

43. Lécureuil $\mathrm{C}$, et al. Trapping and apoptosis of novel subsets of memory T lymphocytes expressing CCR6 in the spleen of HIV-infected patients. Blood 109, 3649-3657 (2007).

44. Cummins NW, Badley AD. Mechanisms of HIV-associated lymphocyte apoptosis: 2010. Cell Death Dis 1, e99-e99 (2010).

45. Imbeault M, Ouellet M, Tremblay MJ. Microarray study reveals that HIV-1 induces rapid type-I interferon-dependent p53 mRNA up-regulation in human primary CD4+ T cells. Retrovirology 6, 5-5 (2009). 
46. Shi B, et al. Inhibition of HIV early replication by the $\mathrm{p} 53$ and its downstream gene $\mathrm{p} 21$. Virology journal 15, 53-53 (2018).

47. Hegedus A, Kavanagh Williamson $M$, Huthoff H. HIV-1 pathogenicity and virion production are dependent on the metabolic phenotype of activated CD4+ T cells. Retrovirology 11, 98-98 (2014).

48. Palmer CS, et al. Increased glucose metabolic activity is associated with CD4+ T-cell activation and depletion during chronic HIV infection. AIDS (London, England) 28, 297309 (2014).

49. Warburg O, Wind F, Negelein E. THE METABOLISM OF TUMORS IN THE BODY. J Gen Physiol 8, 519-530 (1927).

50. Shytaj IL, et al. Glycolysis downregulation is a hallmark of HIV-1 latency and sensitizes infected cells to oxidative stress. EMBO Mol Med 13, e13901 (2021).

51. Deeks SG, Tracy R, Douek DC. Systemic effects of inflammation on health during chronic HIV infection. Immunity 39, 633-645 (2013).

52. Massanella $\mathrm{M}$, Fromentin $\mathrm{R}$, Chomont $\mathrm{N}$. Residual inflammation and viral reservoirs: alliance against an HIV cure. Current opinion in HIV and AIDS 11, 234-241 (2016).

53. Pinzone MR, et al. Longitudinal HIV sequencing reveals reservoir expression leading to decay which is obscured by clonal expansion. Nature Communications 10, 728 (2019).

54. Venanzi Rullo $\mathrm{E}$, et al. Persistence of an intact HIV reservoir in phenotypically naive T cells. JCl Insight 5, (2020).

55. Cros J, et al. Human CD $14<$ sup $>$ dim $</$ sup $>$ Monocytes Patrol and Sense Nucleic Acids and Viruses via TLR7 and TLR8 Receptors. Immunity 33, 375-386 (2010).

56. Wong ME, Jaworowski A, Hearps AC. The HIV Reservoir in Monocytes and Macrophages. Frontiers in immunology 10, (2019).

57. Roederer M, Nozzi JL, Nason MC. SPICE: exploration and analysis of post-cytometric complex multivariate datasets. Cytometry A 79, 167-174 (2011).

58. Vicenti I, et al. Development of an internally controlled quantitative PCR to measure total cell-associated HIV-1 DNA in blood. Clin Chem Lab Med 56, e75-e77 (2018). 
697 Figures and legends

698 Table 1: Patient characteristics

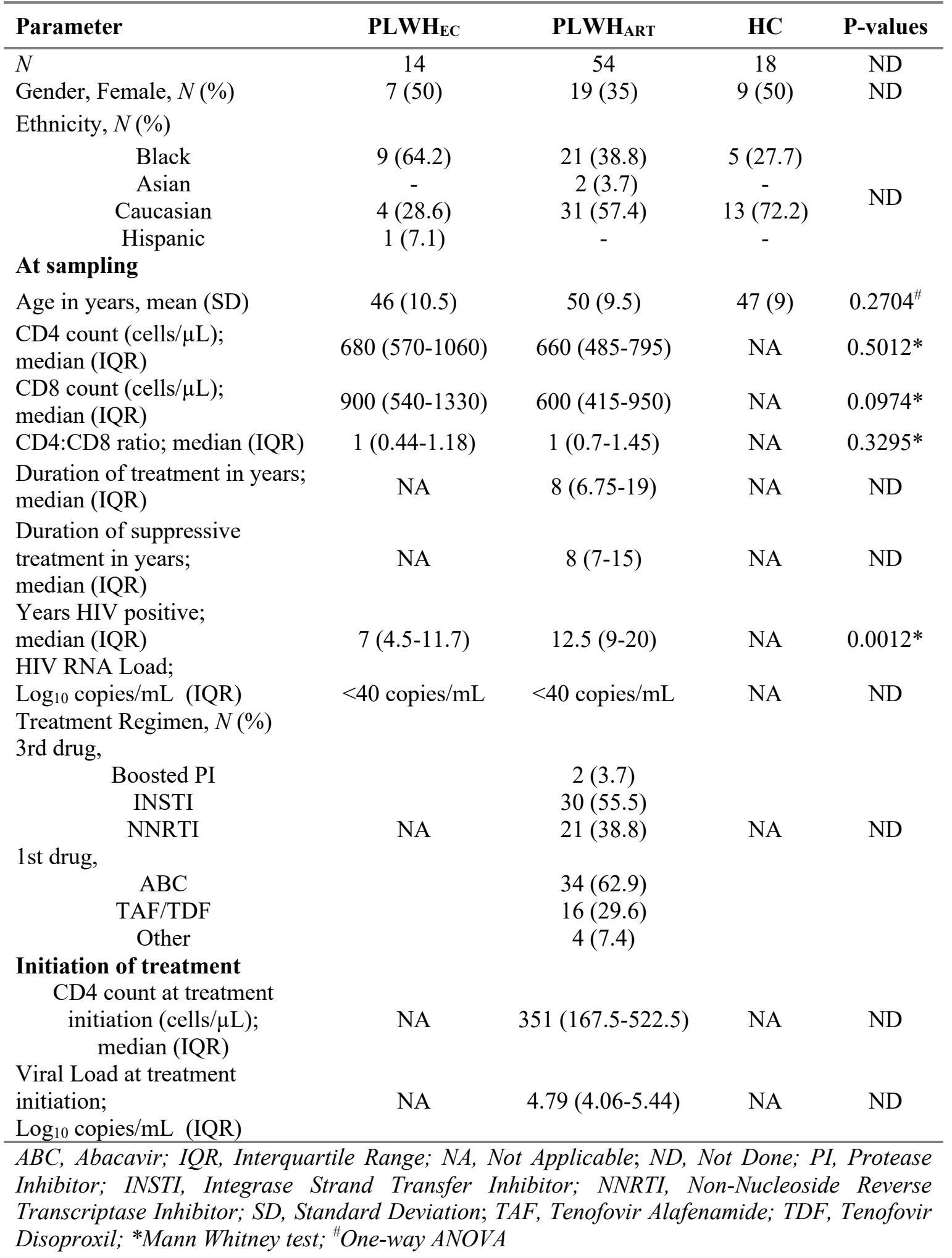


a.

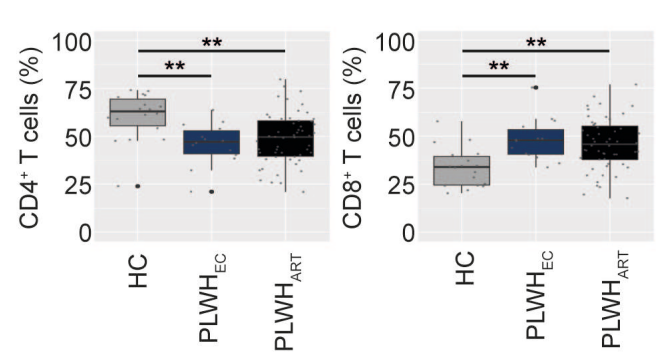

C.
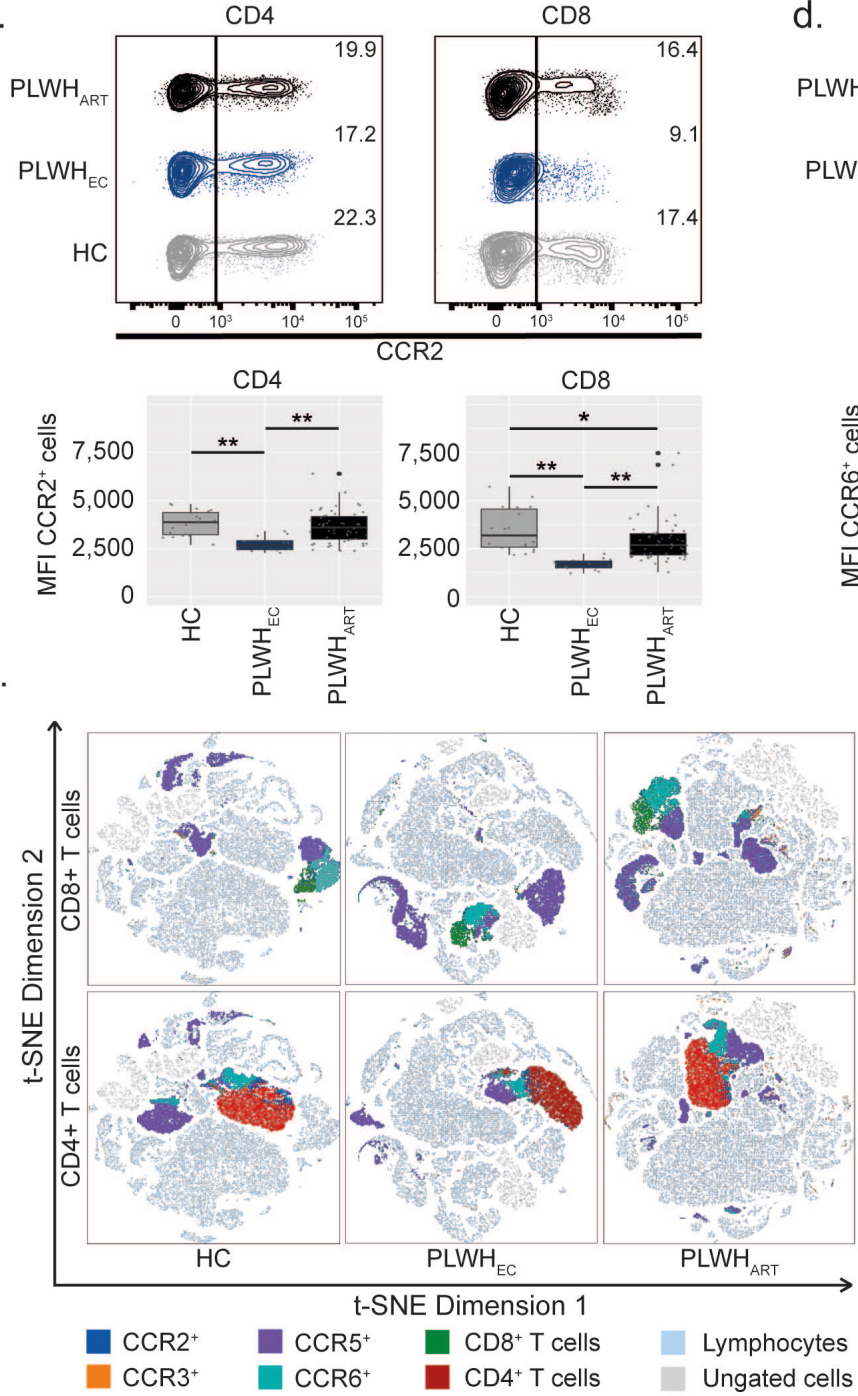

b.
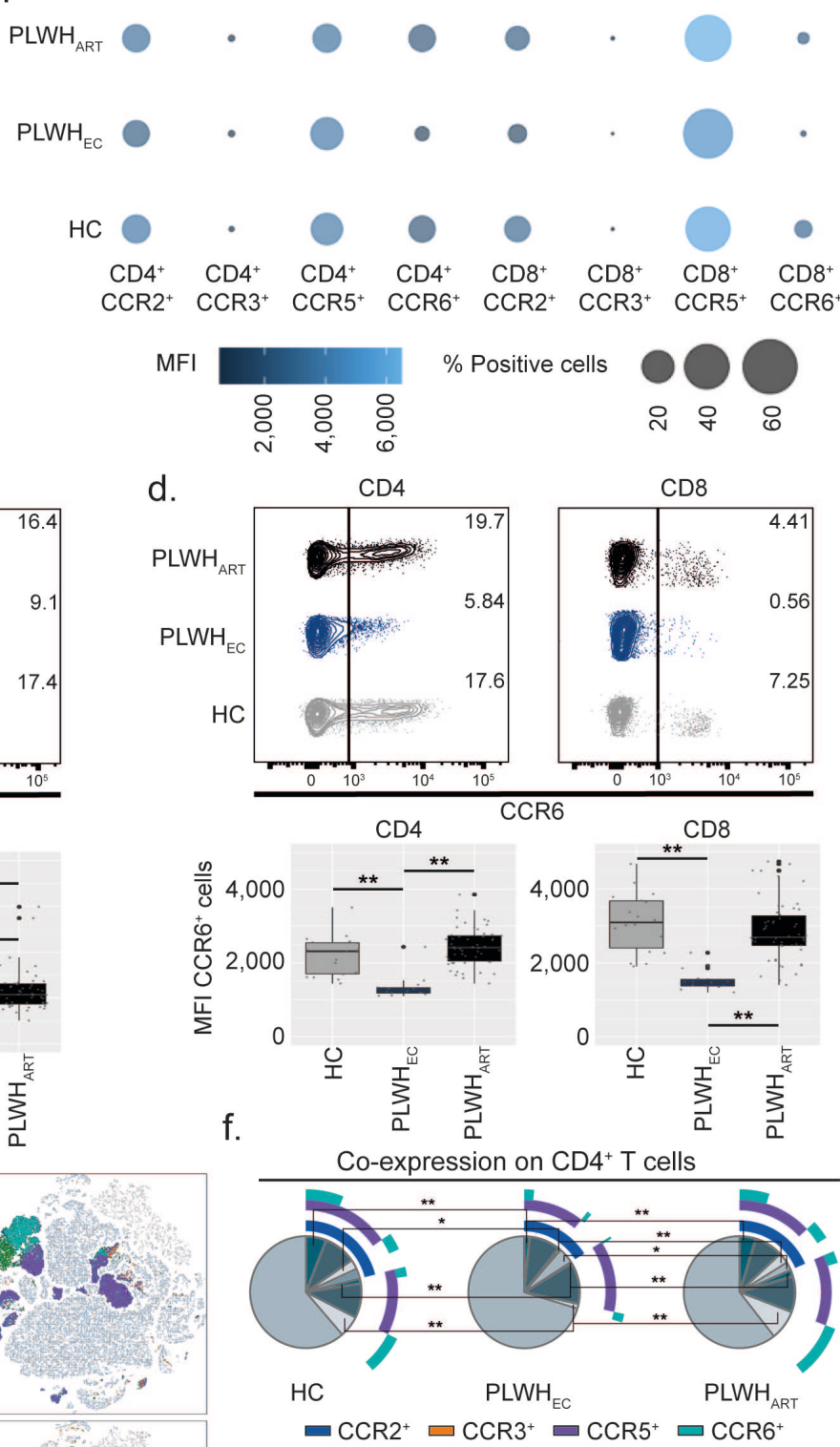

g.

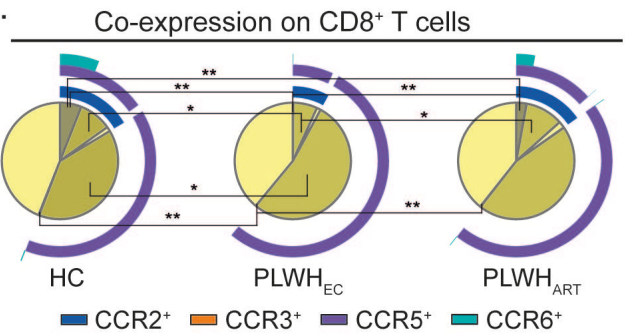

Figure 1: CCR2 and CCR6 are specifically depleted from T lymphocytes in PLWHEC. Receptor

701 expression on $\mathrm{CD}^{+}$and $\mathrm{CD}^{+} \mathrm{T}$ lymphocytes from PLWHEC $(n=14), \mathrm{PLWH}_{\mathrm{ART}}(n=54)$, and HC $(n=18)$. a Frequencies of $\mathrm{CD}^{+} \mathrm{T}$ cells and $\mathrm{CD} 8^{+} \mathrm{T}$ cells in total peripheral blood mononuclear cells. $\mathbf{b}$ Bubble chart representing receptor expression of CCR2, CCR3, CCR5, and CCR6 on CD4 ${ }^{+}$and $\mathrm{CD} 8^{+}$

704 T cells. Size of the bubble corresponds to percentage positive cells while colour represents the median

705 fluorescence intensity (MFI). c CCR2 receptor expression on $\mathrm{CD}^{+}$and $\mathrm{CD} 8^{+} \mathrm{T}$ cells (\% cells and MFI). d CCR6 receptor expression on $\mathrm{CD}^{+}{ }^{+}$and $\mathrm{CD} 8^{+} \mathrm{T}$ cells $(\%$ cells and MFI). e t-SNE plots showing 
707 receptor expression distribution on $\mathrm{CD}^{+}$and $\mathrm{CD} 8^{+} \mathrm{T}$ cells. $\mathrm{f}$ Co-receptor expression of CCR2, CCR3, 708 CCR5, and CCR6 on CD4 ${ }^{+}$T cells. $g$ Co-receptor expression of CCR2, CCR3, CCR5, and CCR6 on $709 \mathrm{CD}^{+} \mathrm{T}$ cells. $\mathbf{c}$ and $\mathbf{d}$ Contour plots show a representing sample corresponding to the median $\%$ of cells 710 within each group. a, c, d, f, $\mathbf{g}$ Statistical significance was determined using two-tailed Mann-Whitney 711 U-test (significance level $p<0.05$, with $*<0.05, * *<0.001$ ) and represented as pie charts or with median 712 using $95 \%$ CI. 
a.

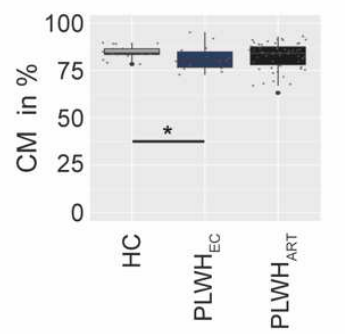

b.

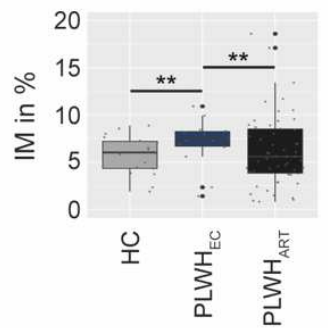

d.
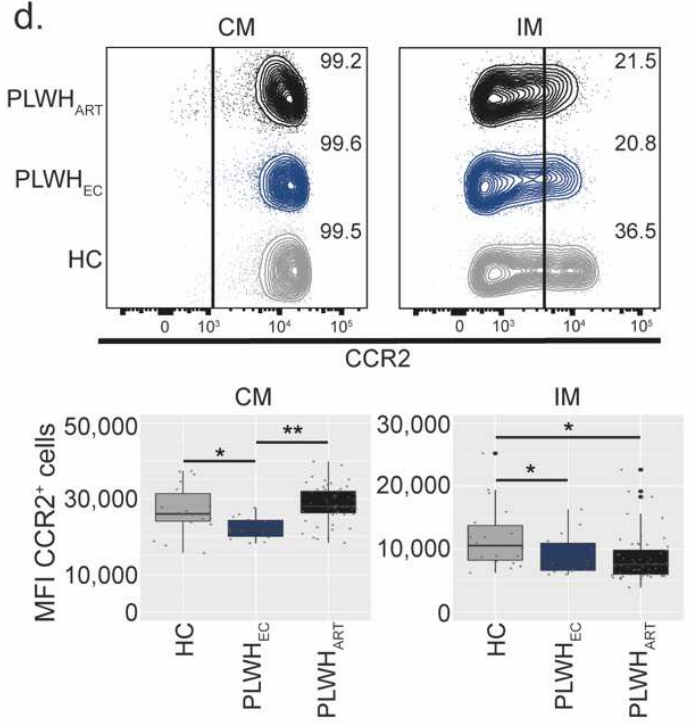

f.

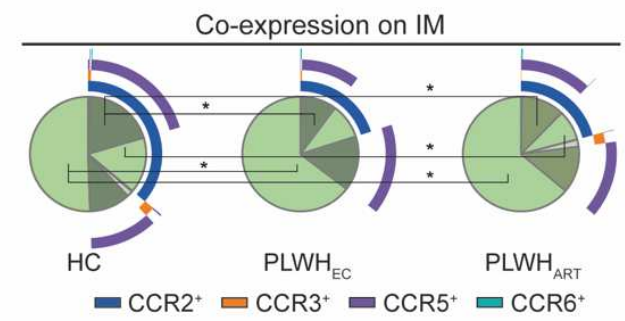

C.

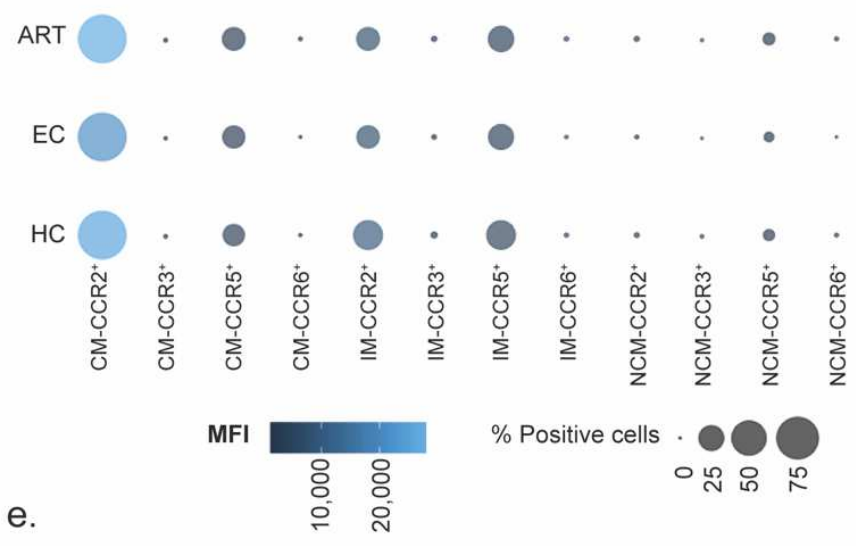

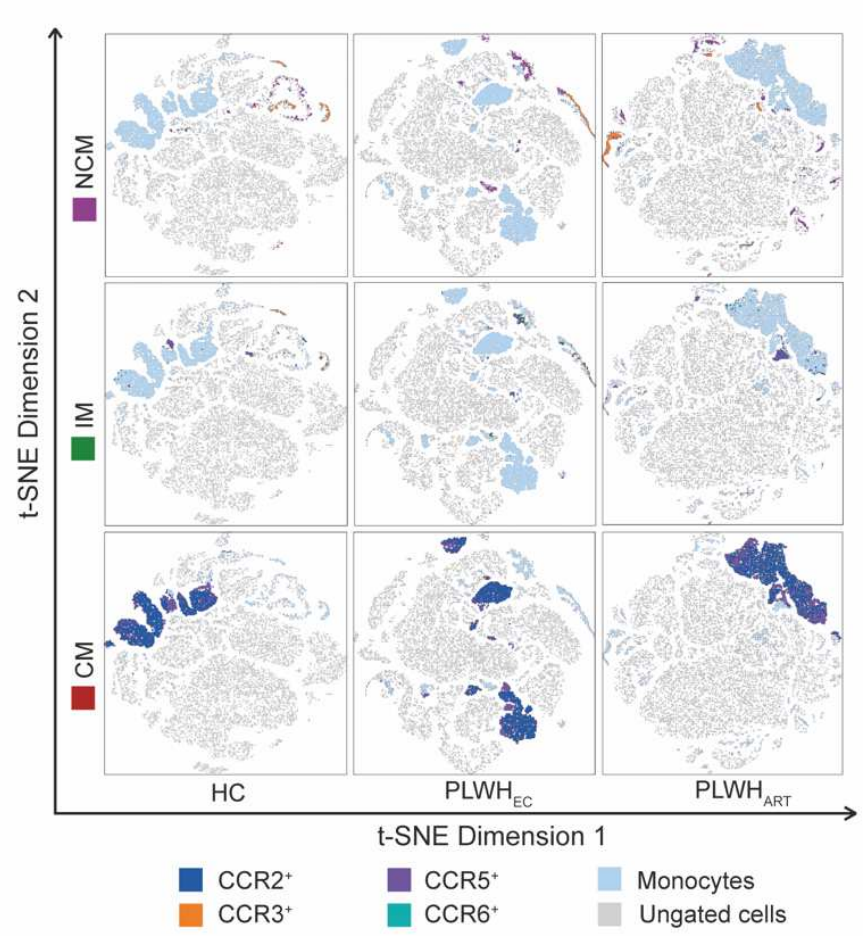

Figure 2: CCR2 is depleted from intermediate monocytes of PLWH. Receptor expression on monocytic subpopulations (classical monocytes (CM); $\mathrm{CD} 14^{+} \mathrm{CD} 16^{-}$, intermediate monocytes (IM) $\mathrm{CD} 14^{+} \mathrm{CD} 16^{+}$, and non-classical monocytes $\left.(\mathrm{NCM}) ; \mathrm{CD}^{-} \mathrm{CD} 16^{+}\right)$in $\mathrm{PLWH}_{\mathrm{EC}}(n=14), \mathrm{PLWH}_{\mathrm{ART}}$ $(n=54)$, and HC $(n=18)$. a, b Percentages of CM (a) and IM (b) from peripheral blood mononuclear cells. c Bubble chart representing receptor expression of CCR2, CCR3, CCR5, and CCR6 on CM, IM, and NCM. Size of the bubble corresponds to percentage positive cells while colour represents the median fluorescent intensity (MFI). d CCR2 receptor expression on CM and IM (\% cells and MFI). e tSNE plots showing receptor expression distribution of CCR2, CCR3, CCR5, and CCR6 on CM, IM, and NCM. f Co-receptor expression of CCR2, CCR3, CCR5, and CCR6 on IM. d Contour plot show a representing sample corresponding to the median $\%$ of cells within each group. a, b, d, f Statistical significance was determined using two-tailed Mann-Whitney U-test (significance level $p<0.05$, with * $<0.05, * *<0.001)$ and represented as pie charts or with median using $95 \% \mathrm{CI}$. 


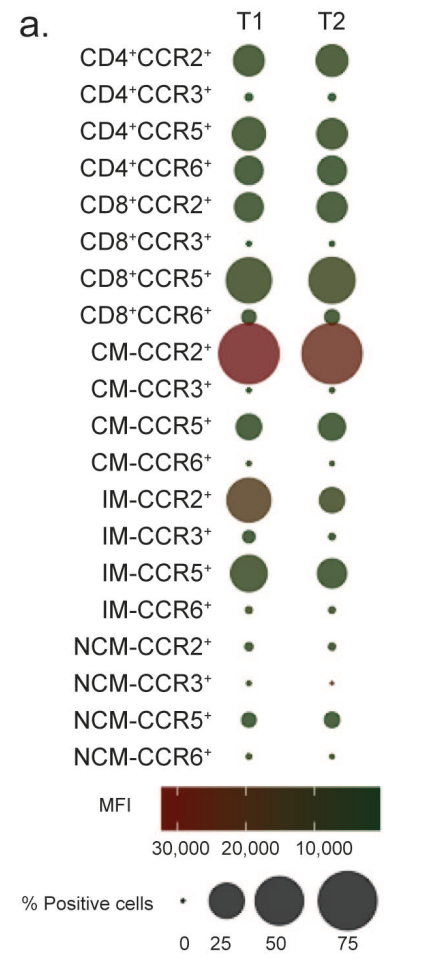

e.

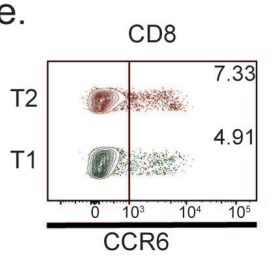

f.

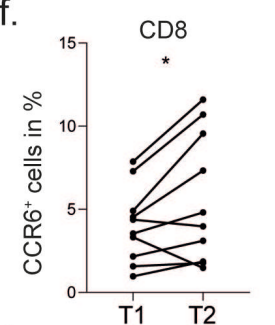

g.

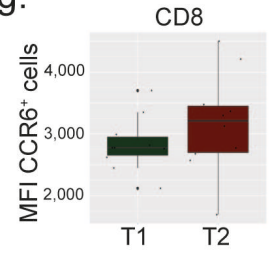

b.
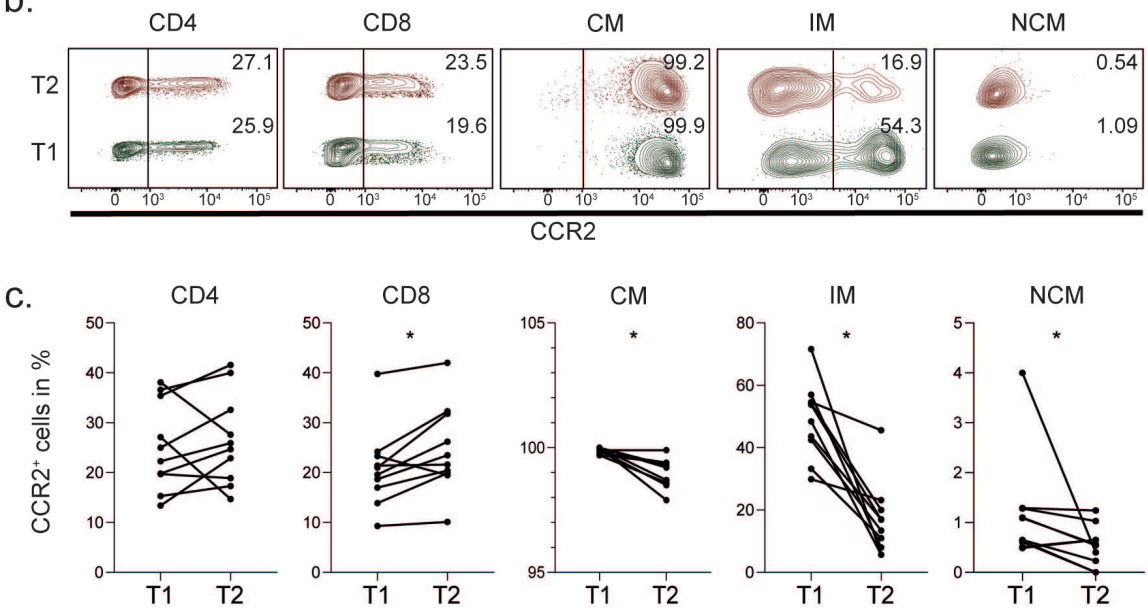

d. $\quad$ CD4
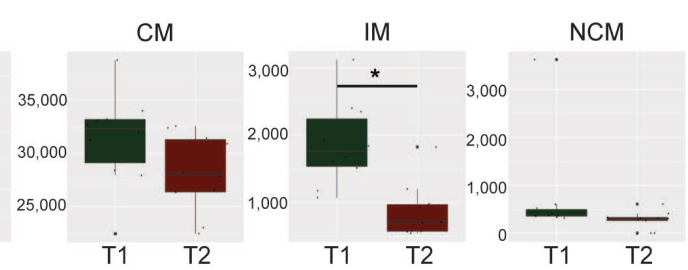

h.

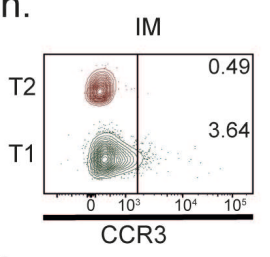

i.

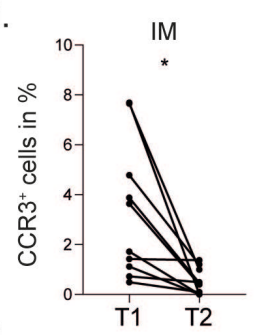

IM

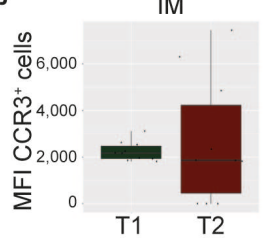

k.

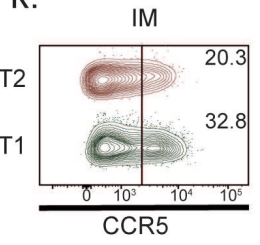

I.
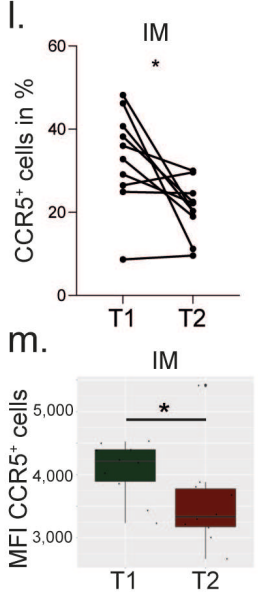

n.

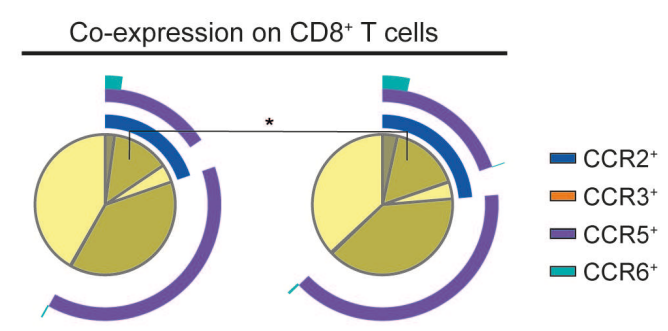

o.

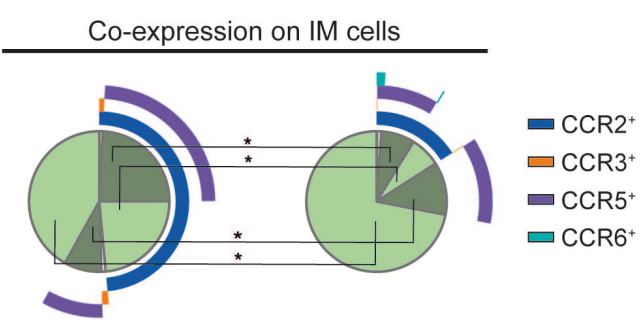

Figure 3: In time, CCR2 and CCR6 increases on CD8 cells but decreases from monocytes in

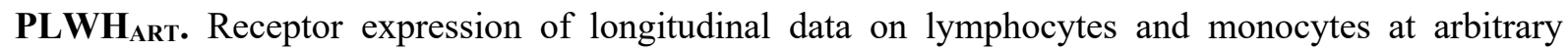
timepoint 1 (T1) $(n=10)$ and three years later at timepoint 2 (T2) $(n=10)$ from total peripheral blood mononuclear cells. a Bubble chart representing receptor expression of CCR2, CCR3, CCR5, and CCR6 on $\mathrm{CD}^{+}$and $\mathrm{CD} 8^{+} \mathrm{T}$ cells together with classical $\left(\mathrm{CM} ; \mathrm{CD} 14^{-} \mathrm{CD} 16^{+}\right)$, intermediate (IM; CD14 $\left.{ }^{-} \mathrm{CD} 16^{+}\right)$, and non-classical (NCM; CD14-CD16 ${ }^{+}$monocytes. Size of the bubble corresponds to percentage positive cells while colour represents the median fluorescent intensity (MFI). b, $\mathbf{c}$ Receptor expression of CCR2 on $\mathrm{CD}^{+}, \mathrm{CD} 8^{+}, \mathrm{CM}$, IM, and NCM. d MFI of CCR2 on $\mathrm{CD} 4^{+}, \mathrm{CD} 8^{+}, \mathrm{CM}$, IM, and NCM. e- 
737 expression of CCR3 on IM. $\mathbf{j}$ MFI of CCR 3 on IM. $\mathbf{k}, \mathbf{1}$ Receptor expression of CCR5 on IM. $\mathbf{m}$ MFI of 738 CCR5 on IM n, o Co-receptor expression of CCR2, CCR3, CCR5, and CCR6 on CD8 ${ }^{+}$T cells (n) and 739 IM (o). b, e, h, $\mathbf{k}$ Contour plots representing the median \% of cells within each group. $\mathbf{c}, \mathbf{d}, \mathbf{f}, \mathbf{g}, \mathbf{i}, \mathbf{j}, \mathbf{l}-\mathbf{o}$ 740 Statistical significance was determined using two-tailed Wilcoxon matched-pairs signed rank test 741 (significance level $p<0.05$, with $*<0.05, * *<0.001$ ) and represented as paired before and after plot, 742 median using $95 \% \mathrm{CI}$ or as pie charts. 
a.

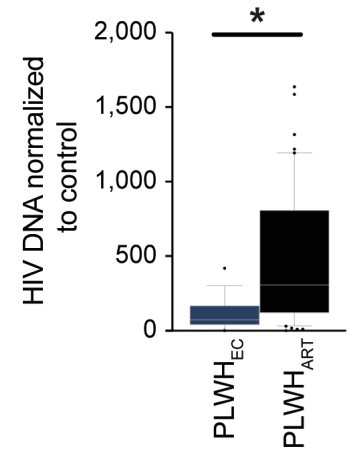

b.

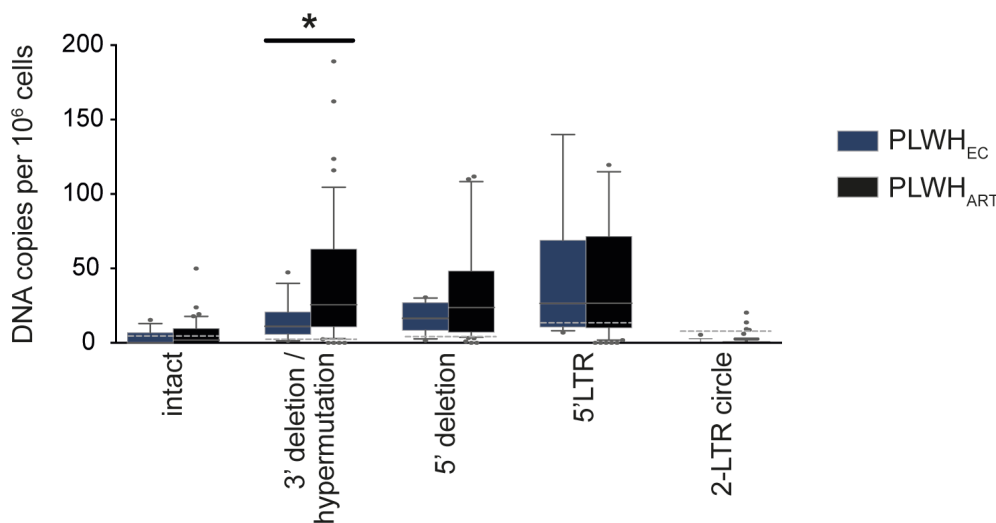

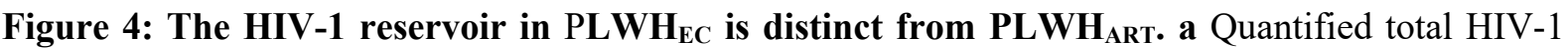
DNA in peripheral blood mononuclear cells from PLWHEC $(n=14)$ and $\operatorname{PLWH}_{\mathrm{ART}}(n=54)$. b Box and Whisker plot shows the distribution of integrated HIV-1 DNA copies per $10^{6}$ PBMCs in PLWH $\mathrm{ART}_{\mathrm{A} T}$ $748 \quad(n=54)$ and $\mathrm{PLWH}_{\mathrm{EC}}(n=14)$ with whiskers representing the 10-90 percentile. The median is represented 749 by the line inside the box. The dotted line marks the background represented by HC samples. Statistical 750 significance was determined using two-tailed Mann-Whitney U-test (significance level $p<0.05$, with * $751<0.05, * *<0.001)$ and represented as median using 95\% CI. 
a.

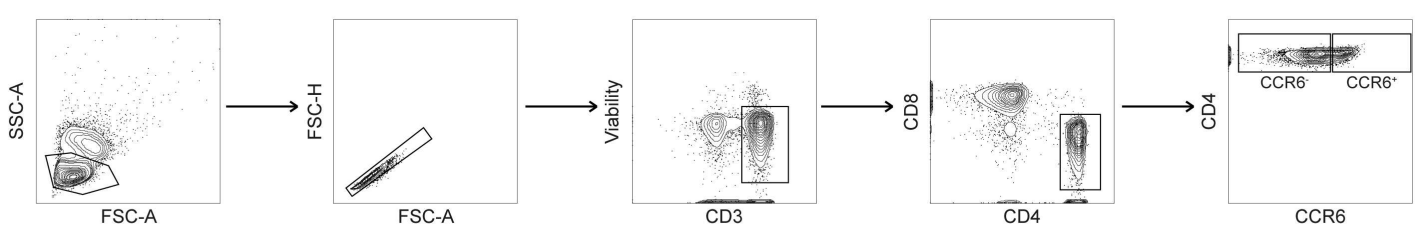

b.
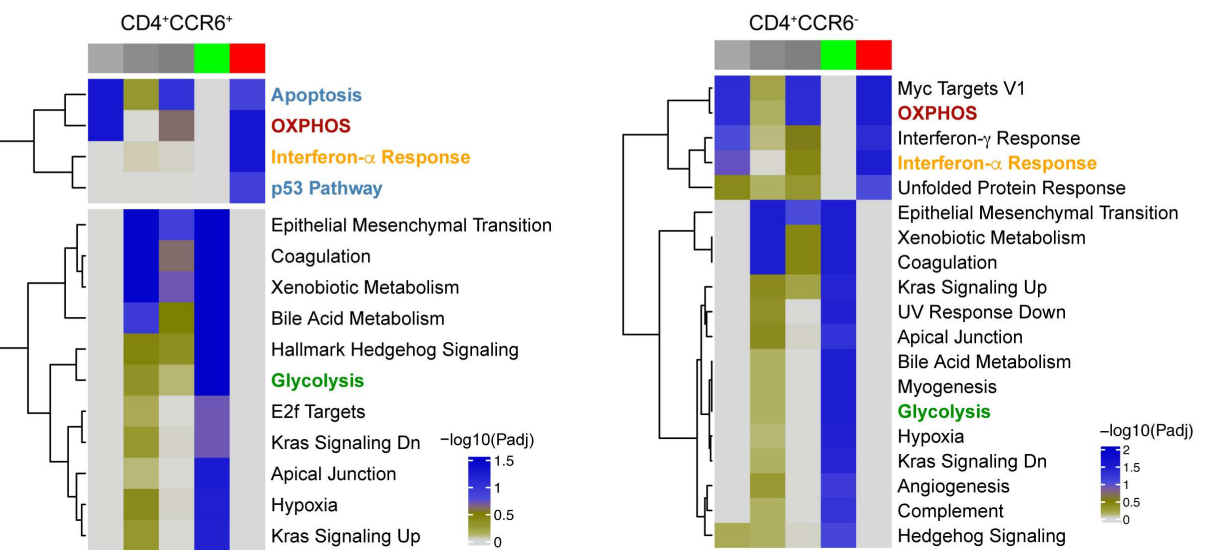

Mixed directional up

Mixed directional down

on-directional

Distinct directional down

Distinct directional up

d.

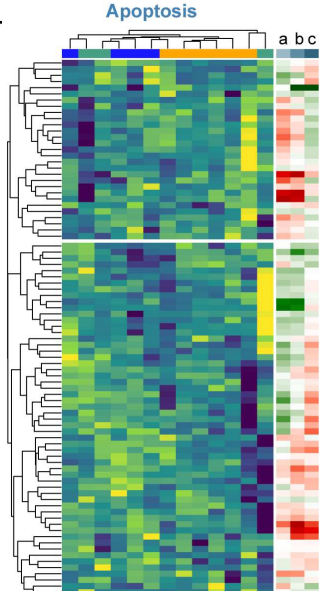

\begin{tabular}{lccl} 
Cohorts & Z-Score (Apoptosis) & Z-Score (p53) & Comparisons \\
ART & 2 & 2 & LFC \\
ART & -1 & 1 & a)ART vs HC \\
EC & -0 & 0 & bECC vs HC \\
HC & -1 & -1 & c)EC vs ART \\
\hline & -1 & -1 &
\end{tabular} e.

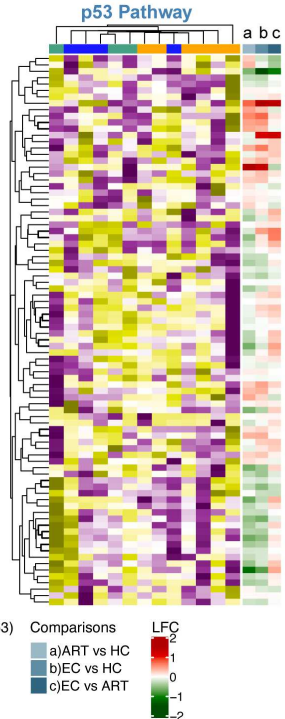

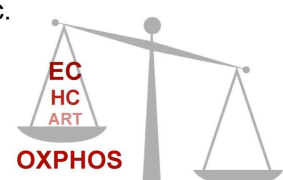

Glycolysis
753

Figure 5: $\mathrm{CD4}^{+} \mathrm{CCR6}^{+}$are enriched in proteins from p53 and apoptosis signalling in $\mathrm{PLWH}_{\mathrm{EC}}$

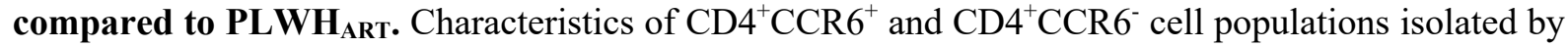
FACS. a Gating strategy for isolation of $\mathrm{CD} 4^{+} \mathrm{CCR} 6^{+}$and $\mathrm{CD} 4^{+} \mathrm{CCR} 6^{-}$cell populations from total peripheral blood mononuclear cells. b Heatmap representation of significant pathways found deregulated in $\mathrm{CD}^{+} \mathrm{CCR}^{+} \mathrm{PLWH}_{\mathrm{EC}}$ compared to $\mathrm{CD}^{+} \mathrm{CCR}^{+} \mathrm{PLWH}_{\mathrm{ART}}$ and in $\mathrm{CD}^{+} \mathrm{CCR} 6^{-} \mathrm{PLWH}_{\mathrm{EC}}$ compared to $\mathrm{CD}^{+}{ }^{+} \mathrm{CCR} 6^{-} \mathrm{PLWH} \mathrm{ART}_{\mathrm{A} T}$ using MSigDBv. Colour gradient corresponds to the negative log scaled adjusted p-values. Each column represents p-values of various directionality classes, calculated for the pathways. Non-directional p-values are calculated based on gene-level statistics regardless of the direction of expression. Mixed directional up and mixed directional down p-values are calculated using the subset of the gene statistics that are up-regulated and down-regulated, respectively. Distinct directional up and distinct directional down p-values are calculated from gene statistics with expression direction. $\mathbf{c}$ Schematic representation of metabolic trade-off between OXPHOS and glycolysis detected 
766 in the cells. d, e Heatmap representation of proteins part of apoptosis (d) and p53 pathway (e). Colour 767 gradient corresponds to the z-score scaled normalized expression values. Column annotation represents 768 the cohorts and row annotation visualizes the $\log 2$ transformed fold change values in each of the 769 differential expression analysis of $\mathrm{PLWH}_{\mathrm{ART}}$ compared with $\mathrm{HC}, \mathrm{PLWH}_{\mathrm{EC}}$ compared with $\mathrm{HC}$ and 770 PLWH $_{\mathrm{EC}}$ compared with PLWH $\mathrm{ART}_{\text {. }}$ f Network visualization of significant pathways enriched in $771 \mathrm{CD}^{+} \mathrm{CCR} 6^{+} \mathrm{PLWH}_{\mathrm{EC}}$ compared to $\mathrm{CD} 4^{+} \mathrm{CCR} 6^{+} \mathrm{PLWH}_{\mathrm{ART}}$. Each edge represents association of the 772 protein with the corresponding pathways. Circular nodes denote proteins and square shaped nodes are 773 pathways. Red colour gradient and green colour gradient represent up-regulation and down-regulation 774 of the proteins in $\mathrm{CD} 4^{+} \mathrm{CCR} 6^{+} \mathrm{PLWH}_{\mathrm{EC}}$ compared to $\mathrm{CD} 4^{+} \mathrm{CCR} 6{ }^{+} \mathrm{PLWH}_{\mathrm{ART}}$. 


\section{Supplementary Files}

This is a list of supplementary files associated with this preprint. Click to download.

- SupplementaryTablesandFiguresSSA.docx 